\title{
Article
}

\section{Higer-order commutators of parametrized Marcinkewicz integrals on Herz spaces with variable exponent}

\author{
Omer Abdalrhman ${ }^{1, *}$, Afif Abdalmonem ${ }^{2}$ and Shuangping Tao ${ }^{3}$ \\ College of Education, Shendi University, Shendi, River Nile State, Sudan. \\ 2 Faculty of Science, University of Dalanj, Dalanj, South kordofan, Sudan. \\ 3 College of Mathematics and Statistics, Northwest Normal University, Lanzhou, Gansu, P.R. China. \\ * Correspondence: humoora@gmail.com
}

Received: 10 October 2019; Accepted: 15 March 2020; Published: 31 March 2020.

Abstract: Let $0<\rho<n$ and $\mu_{\Omega}^{\rho}$ be the Parametrized Marcinkiewicz integrals operator. In this work, the bondedness of $\mu_{\Omega}^{\rho}$ is discussed on Herz spaces $\dot{K}_{p(\cdot)}^{\alpha, q(\cdot)}\left(\mathbb{R}^{n}\right)$, where the two main indices are variable exponent. The boundedness of the commutators generated by BOM function, Lipschitz function and parametrized Marcinkiewicz integrals operator is also discussed.

Keywords: BMO function, Commutator, Herz space with variable exponent, Lipschitz function, Parametrized Marcinkiewicz integral operator.

\section{Introduction}

uppose $\mathbb{S}^{n-1}$ for $n \geq 2$ is the unit sphere in $\mathbb{R}^{n}$ equipped with the normalized Lebesgue measure $\mathrm{d} \sigma$. Further suppose that $\Omega$ is a homogeneous function of degree zero on $\mathbb{R}^{n}$ satisfying $\Omega \in L^{1}\left(\mathbb{S}^{n-1}\right)$ and

$$
\int_{\mathbb{S}^{n-1}} \Omega\left(x^{\prime}\right) \mathrm{d} \sigma\left(x^{\prime}\right)=0, \text { where } x^{\prime}=x /|x|(x \neq 0) .
$$

For $0<\rho<n$, the parametrized Marcinkiewicz integrals is defined as;

$$
\mu_{\Omega}^{\rho}(h)(x)=\left(\int_{0}^{\infty}\left|F_{\Omega, t}^{\rho}(h)(x)\right|^{2} \frac{\mathrm{d} t}{t^{2 \rho+1}}\right)^{1 / 2},
$$

where $F_{\Omega, t}^{\rho}(h)(x)=\int_{|x-y| \leq t} \frac{\Omega(x-y)}{|x-y|^{n-\rho}} h(y) \mathrm{d} y, t>0$.

For $m \in \mathbb{N}, b \in \mathrm{BMO}\left(\mathbb{R}^{n}\right)$, the higher-order commutator of parametrized Marcinkiewicz integral is defined as;

$$
\left[b^{m}, \mu_{\Omega}^{\rho}\right](h)(x)=\left(\int_{0}^{\infty}\left|\int_{|x-y| \leq t} \frac{\Omega(x-y)}{|x-y|^{n-\rho}}[b(x)-b(y)]^{m} h(y) \mathrm{d} y\right|^{2} \frac{d t}{t^{2 \rho+1}}\right)^{1 / 2}, t>0 .
$$

It is easy to see that when $\rho=1$, and $\mu^{\rho}(h)=\mu^{1}(h)$, then (2) is the classical Marcinkiewicz integral $\mu(h)$ introduced by Stein in [1]. It has been proved in [1] that if $\Omega \in \operatorname{Lip}_{\gamma}\left(\mathbb{S}^{n-1}\right)(0<\gamma \leq 1)$ and $\Omega$ is continuous, then the operator $\mu(h)$ is of the type $(q, q)$ for $1<q \leq 2$ and of the weak type $(1,1)$. Benedek et al., [2] proved that if $\Omega \in C^{1}\left(\mathbb{S}^{n-1}\right)$, then $\mu(h)$ it is of type $(q, q)$ for any $1<q \leq \infty$. The $L^{p}$ boundedness of the $\mu(h)$ has been studied in $[1,3-5]$.

In 1960, Hörmander [4] introduced the parametrized Marcinkiewicz integral operators proved that if $\Omega \in \operatorname{Lip}_{\gamma}\left(\mathbb{S}^{n-1}\right), 0<\gamma \leq 1$, then it is of strong type $(q, q)$ for $1<q \leq 2$. Sakamoto and Yabuta [6] proved the boundedness of the operator $\mu^{\rho}(h)$ on $L^{q}\left(\mathbb{R}^{n}\right)$. Shi and Jiang [7] considered the weighted $L^{q}$-boundedness of parametrized Marcinkiewicz integral operator and its higher order commutator. Note that the Littlewood-paley $g$-function played very important roles in harmonic analysis and the parameterized Marcinkiewick integral is a special case of the Littlewood-paley $g$-function. Many authors studied properties of $\mu^{\rho}(h)$ on different function spaces, for examples [8-14]. 
In the last three decade, the generalized Orlicz-Lebesgue spaces and the corresponding generalized Orlicz-Sobolev spaces have been extensively studied by many researchers. The variable Lebesgue spaces are special cases of generalized orliz spaces which introduced by Nakano in [15] and developed in [16,17]. In addition, for properties of $L^{p(\cdot)}$ spaces we refer to [18-20], and the fundamental paper of Kováčik and Rákosník [21] appeared in 1990. By virtue of this works many function spaces appeared [22-25]. Recently, in 2015, Lijuan and Tao established the Herz spaces with two variable exponents $p(\cdot), q(\cdot)$ in the paper [26].

The main purpose of this work is to discuss the boundedness of parameterized Marcinkiewicz integral and it's higher order commutators with rough kernels on Herz spaces with two variable exponents. The boundedness of higher order commutator generated by BOM function and parameterized Marcinkiewicz integral is also obtained.

Let $Y$ be a measurable set in $\mathbb{R}^{n}$ with $|Y|>0$.

Definition 1. Let $p(\cdot): \mathrm{Y} \rightarrow[1, \infty)$ be a measurable function. The Lebesgue space with variable exponent $L^{p(\cdot)}(\mathrm{Y})$ is defined by

$$
L^{p(\cdot)}(\mathrm{Y})=\left\{h \text { is measurable }: \int_{\Omega}\left(\frac{|h(x)|}{\eta}\right)^{p(x)} d x<\infty \text { for some constant } \eta>0\right\}
$$

The space $L_{l o c}^{p(\cdot)}(\mathrm{Y})$ is defined by

$$
L_{\text {loc }}^{p(\cdot)}(\mathrm{Y})=\left\{\mathrm{h} \text { is measurable }: h \in L^{p(\cdot)}(\mathrm{K}) \text { for all compact } K \subset \mathrm{Y}\right\}
$$

The Lebesgue spaces $L^{p(\cdot)}(\mathrm{Y})$ is a Banach spaces with the norm defined by

$$
\|h\|_{L^{p(\cdot)}(\mathrm{Y})}=\inf \left\{\eta>0: \int_{\Omega}\left(\frac{|h(x)|}{\eta}\right)^{p(x)} d x \leq 1\right\}
$$

We denote

$$
p_{-}=\operatorname{essinf}\{p(x): x \in \mathrm{Y}\}, \quad p_{+}=\operatorname{ess} \sup \{p(x): x \in \mathrm{Y}\},
$$

then $\mathcal{P}(\mathrm{Y})$ consists of all $p(\cdot)$ satisfying $p_{-}>1$ and $p_{+}<\infty$.

Let $M$ be the Hardy-Littlewood maximal operator. We denote $\mathcal{B}(\mathrm{Y})$ to be the set of all function $p(\cdot) \in$ $\mathcal{P}(\mathrm{Y})$ such that $M$ is bounded on $L^{p(\cdot)}(\mathrm{Y})$.

Now, let us recall the definition of Herz spaces with variable exponents.

Definition 2. [26]Let $\alpha \in \mathbb{R}^{n}, q(\cdot), p(\cdot) \in \mathcal{P}\left(\mathbb{R}^{n}\right)$. The homogeneous Herz space with variable exponent $\dot{K}_{p(\cdot)}^{\alpha, q(\cdot)}\left(\mathbb{R}^{n}\right)$ is defined by

$$
\dot{K}_{p(\cdot)}^{\alpha, q(\cdot)}\left(\mathbb{R}^{n}\right)=\left\{h \in L_{l o c}^{p(\cdot)}\left(\mathbb{R}^{n} \backslash\{0\}\right):\|h\|_{\dot{K}_{p(\cdot)}^{\alpha, q(\cdot)}\left(\mathbb{R}^{n)}\right.}<\infty\right\},
$$

where

$$
\|h\|_{\dot{K}_{p_{(\cdot)}^{\alpha}(\cdot)}^{\alpha(\cdot)}}=\left\|\left\{2^{k \alpha}\left|h \chi_{k}\right|\right\}_{k=0}^{\infty}\right\|_{l^{q}(\cdot)\left(L^{p(\cdot)}\right)}=\inf \left\{\eta>0: \sum_{k=-\infty}^{\infty}\left\|\left(\frac{2^{k \alpha}\left|h \chi_{k}\right|}{\eta}\right)^{q(\cdot)}\right\|_{L^{\frac{p(\cdot)}{q(\cdot)}}} \leq 1\right\} .
$$

Remark 1. Let $v \in \mathbb{N}, a_{v} \geq 0,1 \leq p_{v}<\infty$, then

$$
\sum_{v=0}^{\infty} a_{v} \leq\left(\sum_{v=0}^{\infty} a_{v}\right)^{p_{*}}
$$


where

$$
p_{*}= \begin{cases}\min _{v \in \mathbb{N}} p_{v}, & \sum_{v=0}^{\infty} a_{v} \leq 1 \\ \max _{v \in \mathbb{N}} p_{v}, & \sum_{v=0}^{\infty} a_{v}>1 .\end{cases}
$$

\section{Remark 2. [26]}

1. If $q_{1}(\cdot), q_{2}(\cdot) \in \mathcal{P}\left(\mathbb{R}^{n}\right)$ satisfying $\left(q_{1}\right)_{+} \leq\left(q_{2}\right)_{+}$, then $K_{p(\cdot)}^{\alpha, q_{1}(\cdot)}\left(\mathbb{R}^{n}\right) \subset K_{p(\cdot)}^{\alpha, q_{2}(\cdot)}\left(\mathbb{R}^{n}\right), \quad \dot{K}_{p(\cdot)}^{\alpha, q_{1}(\cdot)}\left(\mathbb{R}^{n}\right) \subset$ $\dot{K}_{p(\cdot)}^{\alpha, q_{2}(\cdot)}\left(\mathbb{R}^{n}\right)$

2. If $q_{1}(\cdot), q_{2}(\cdot) \in \mathcal{P}\left(\mathbb{R}^{n}\right)$ and $\left(q_{1}\right)_{+} \leq\left(q_{2}\right)_{-}$, then $\frac{q_{2}(\cdot)}{q_{1}(\cdot)} \in \mathcal{P}\left(\mathbb{R}^{n}\right)$ and $\frac{q_{2}(\cdot)}{q_{1}(\cdot)} \geq 1$.

By Remark 1 , for any $h \in \dot{K}_{p(\cdot)}^{\alpha, q(\cdot)}\left(\mathbb{R}^{n}\right)$, we have

$$
\sum_{k=-\infty}^{\infty}\left\|\left(\frac{2^{k \alpha}\left|h \chi_{k}\right|}{\eta}\right)^{q_{2}(\cdot)}\right\|_{L^{\frac{p(\cdot)}{q_{2}(\cdot)}}} \leq \sum_{k=-\infty}^{\infty}\left\|\left(\frac{2^{k \alpha}\left|h \chi_{k}\right|}{\eta}\right)^{q_{1}(\cdot)}\right\|_{L^{\frac{p(\cdot)}{q_{1}(\cdot)}}}^{p_{v}} \leq\left\{\sum_{k=-\infty}^{\infty}\left\|\left(\frac{2^{k \alpha}\left|h \chi_{k}\right|}{\eta}\right)^{q_{1}(\cdot)}\right\|_{L^{\frac{p(\cdot)}{q_{1}(\cdot)}}}^{p_{h}}\right\}^{p_{*}} \leq 1 ;
$$

where

$$
p_{v}= \begin{cases}\left(\frac{q_{2}(\cdot)}{q_{1}(\cdot)}\right)_{-}, & \frac{2^{k \alpha}\left|f \chi_{k}\right|}{\eta} \leq 1 \\ \left(\frac{q_{2}(\cdot)}{q_{1}(\cdot)}\right)_{+}, & \frac{2^{k \alpha}\left|f \chi_{k}\right|}{\eta}>1\end{cases}
$$

and

$$
p_{*}= \begin{cases}\min _{v \in \mathbb{N}} p_{v}, & \sum_{v=0}^{\infty} a_{v} \leq 1 \\ \max _{v \in \mathbb{N}} p_{v}, & \sum_{v=0}^{\infty} a_{v}>1\end{cases}
$$

This implies that $\dot{K}_{p(\cdot)}^{\alpha, q_{1}(\cdot)}\left(\mathbb{R}^{n}\right) \subset \dot{K}_{p(\cdot)}^{\alpha, q q_{2}(\cdot)}\left(\mathbb{R}^{n}\right)$. Similarly, we get $K_{p(\cdot)}^{\alpha, q_{1}(\cdot)}\left(\mathbb{R}^{n}\right) \subset K_{p(\cdot)}^{\alpha, q_{2}(\cdot)}\left(\mathbb{R}^{n}\right)$.

Definition 3. For all $0<\gamma \leq 1$, the Lipschitz space $\dot{\Lambda}_{\gamma}\left(\mathbb{R}^{n}\right)$ is defined by

$$
\dot{\Lambda}_{\gamma}\left(\mathbb{R}^{n}\right)=\left\{h:\|h\|_{\dot{\Lambda}_{\gamma}\left(\mathbb{R}^{n}\right)}=\sup _{x, y \in \mathbb{R}^{n} ; x \neq y} \frac{|h(x)-h(y)|}{|x-y|^{\gamma}}<\infty\right\} .
$$

Definition 4. The BMO function and $\mathrm{BMO}$ norm are defined by

$$
\begin{aligned}
\operatorname{BMO}\left(\mathbb{R}^{n}\right) & :=\left\{b \in L_{l o c}^{1}\left(\mathbb{R}^{n}\right):\|b\|_{\mathrm{BMO}\left(\mathbb{R}^{n}\right)}<0\right\}, \\
\|b\|_{\mathrm{BMO}\left(\mathbb{R}^{n}\right)} & :=\sup _{Q: \text { cube }}|Q|^{-1} \int_{Q}\left|b(x)-b_{Q}\right| \mathrm{d} x .
\end{aligned}
$$

From here, we suppose that $B_{k}=\left\{x \in \mathbb{R}^{n}:|x| \leq 2^{k}\right\}$, and $C_{k}=B_{k} \backslash B_{k-1}, \chi_{k}=\chi_{C_{k}}, k \in \mathbb{Z}$.

\section{Preliminary Lemmas}

Proposition 1. [27] Let a function $p(\cdot): \mathbb{R}^{n} \rightarrow[1, \infty)$. If $p(\cdot) \in \mathcal{P}\left(\mathbb{R}^{n}\right)$ satisfies

$$
|p(x)-p(y)| \leq \frac{-C}{\log (|x-y|)} ; \quad|x-y| \leq 1 / 2,
$$

and

$$
|p(x)-p(y)| \leq \frac{C}{\log (e+|x|)} ; \quad|y| \geq|x|,
$$

then $p(\cdot) \in \mathfrak{B}\left(\mathbb{R}^{n}\right)$. 
Lemma 1. [21] (Generalized Hölder Inequality) Let $p(\cdot), p_{1}(\cdot), p_{2}(\cdot) \in \mathcal{P}\left(\mathbb{R}^{n}\right)$, then

1. for every $h \in L^{p_{1}(\cdot)}\left(\mathbb{R}^{n}\right)$ and $g \in L^{p_{2}(\cdot)}\left(\mathbb{R}^{n}\right)$, we have $\int_{\mathbb{R}^{n}}|h(x) g(x)| d x \leq C\|h\|_{L^{p(\cdot)}\left(\mathbb{R}^{n}\right)}\|g\|_{L^{p^{(\cdot)}\left(\mathbb{R}^{n}\right)}}$, where $C_{p}=1+\frac{1}{p_{-}}-\frac{1}{p_{+}}$

2. for every $h \in L^{p_{1}(\cdot)}\left(\mathbb{R}^{n}\right), g \in L^{p_{2}(\cdot)}\left(\mathbb{R}^{n}\right)$, when $\frac{1}{p(\cdot)}=\frac{1}{p_{2}(\cdot)}+\frac{1}{p_{1}(\cdot)}$, we have $\|h(x) g(x)\|_{L^{p(\cdot)}\left(\mathbb{R}^{n}\right)} \leq$ $C\|g(x)\|_{L^{p_{2}\left(\mathbb{R}^{n}\right)}}\|h(x)\|_{L^{p_{1}(\cdot)}\left(\mathbb{R}^{n}\right)}$, where $C_{p_{1}, p_{2}}=\left[1+\frac{1}{p_{1-}}-\frac{1}{p_{1+}}\right]^{\frac{1}{p_{-}}}$.

Lemma 2. $[18,19]$ Let $p(\cdot) \in \mathcal{B}\left(\mathbb{R}^{n}\right)$. If there exists a positive constants $C, \delta_{1}, \delta_{2}$ such that $\delta_{1}, \delta_{2}<1$, then, for all balls $B \subset \mathbb{R}^{n}$ and all measurable subset $R \subset B$, we have

$$
\frac{\left\|\chi_{R}\right\|_{L^{p(\cdot)}\left(\mathbb{R}^{n}\right)}}{\left\|\chi_{B}\right\|_{L^{p(\cdot)}\left(\mathbb{R}^{n}\right)}} \leq C \frac{|R|}{|B|}, \quad \frac{\left\|\chi_{R}\right\|_{L^{p^{\prime} \cdot(\cdot)}\left(\mathbb{R}^{n}\right)}}{\left\|\chi_{B}\right\|_{L^{p_{u}^{\prime}(\cdot)}\left(\mathbb{R}^{n}\right)}} \leq C\left(\frac{|R|}{|B|}\right)^{\delta_{2}}, \quad \frac{\left\|\chi_{R}\right\|_{L^{p(\cdot)}\left(\mathbb{R}^{n}\right)}}{\left\|\chi_{B}\right\|_{L^{p(\cdot)}\left(\mathbb{R}^{n}\right)}} \leq C\left(\frac{|R|}{|B|}\right)^{\delta_{1}} .
$$

Lemma 3. [28] Let $p(\cdot) \in \mathcal{B}\left(\mathbb{R}^{n}\right)$, then there exists a constant $C>0$ such that for any balls $B$ in $\mathbb{R}^{n}$, we have

$$
\frac{1}{|B|}\left\|\chi_{B}\right\|_{L^{p(\cdot)}\left(\mathbb{R}^{n}\right)}\left\|\chi_{B}\right\|_{L^{p^{\prime}(\cdot)}\left(\mathbb{R}^{n}\right)} \leq C .
$$

Lemma 4. [29] Let $p(\cdot) \in \mathcal{B}\left(\mathbb{R}^{n}\right)$, and $b \in \mathrm{BMO}\left(\mathbb{R}^{n}\right)$. If $i, j \in \mathbb{Z}$ with $i<j$, then we have

1. $C^{-1}\|b\|_{\mathrm{BMO}\left(\mathbb{R}^{n}\right)} \leq \sup _{B} \frac{1}{\left\|\chi_{B}\right\|_{L^{p(\cdot)}\left(\mathbb{R}^{n}\right)}}\left\|\left(b-b_{B}\right) \chi_{B}\right\|_{L^{p(\cdot)}\left(\mathbb{R}^{n}\right)} \leq C\|b\|_{\mathrm{BMO}\left(\mathbb{R}^{n}\right)} ;$

2. $\left\|\left(b-b_{B_{i}}\right) \chi_{B_{j}}\right\|_{L^{q(\cdot)}\left(\mathbb{R}^{n}\right)} \leq C(j-i)\|b\|_{\mathrm{BMO}\left(\mathbb{R}^{n}\right)}\left\|\chi_{B_{j}}\right\|_{L^{q(\cdot)\left(\mathbb{R}^{n}\right)}}$.

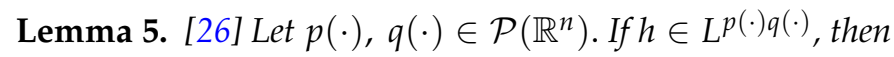

$$
\min \left(\|h\|_{L^{p(\cdot) g(\cdot)}}^{q_{+}},\|h\|_{L^{p(\cdot) q(\cdot)}}^{q_{-}} \leq\left\|\left.|| h\right|^{q(\cdot)}\right\|_{L^{p(\cdot)}} \leq \max \left(\|h\|_{L^{p(\cdot) q(\cdot)}}^{q_{+}},\|h\|_{L^{p(\cdot) q(\cdot)}}^{q_{-}}\right) .\right.
$$

Lemma 6. [30] Let $a>0,0<d \leq s, 1 \leq s \leq \infty$ and $\frac{-s n+(n-1) d}{s}<v<\infty$, then

$$
\left(\int_{|y| \leq a|x|}|y|^{v}|\Omega(x-y)|^{d} d y\right)^{1 / d} \leq C|x|^{(v+n) / d}\|\Omega\|_{L^{s}\left(\mathbb{S}^{n-1}\right)} .
$$

Lemma 7. [31] Let the variable exponent $\tilde{q}(\cdot)$ is defined by $\frac{1}{p(x)}=\frac{1}{\tilde{q}(x)}+\frac{1}{q}\left(x \in \mathbb{R}^{n}\right)$, then we have

$$
\|h g\|_{L^{p(\cdot)}\left(\mathbb{R}^{n}\right)} \leq C\|g\|_{L^{q}\left(\mathbb{R}^{n}\right)}\|h\|_{L^{\tilde{q} \cdot \cdot\left(\mathbb{R}^{n}\right)}} .
$$

Lemma 8. Let $p(\cdot) \in \mathcal{B}\left(\mathbb{R}^{n}\right), \Omega \in L^{s}\left(\mathbb{S}^{n-1}\right)$ and $0<\rho<n$. If there exists a constant $C>0$ independent of $h$, then $\mu_{\Omega}^{\rho}$ is bounded from $L^{p(\cdot)}$ to it self.

Lemma 9. Let $b \in \operatorname{BMO}\left(\mathbb{R}^{n}\right)$ and $m \in \mathbb{N}$. Further let that $p(\cdot) \in \mathcal{B}\left(\mathbb{R}^{n}\right), \Omega \in L^{s}\left(\mathbb{S}^{n-1}\right)$ and $0<\rho<n$. If there exists a constant $C>0$ independent of $h$, then $\left[b^{m}, \mu_{\Omega}^{\rho}\right]$ is bounded from $L^{p(\cdot)}$ to itself.

Lemma 10. Let $b \in \dot{\Lambda}_{\gamma}\left(\mathbb{R}^{n}\right), 0<\gamma \leq 1, m \in \mathbb{N}$ and $0<\rho<n$. If $q_{1}(\cdot) \in \mathcal{P}\left(\mathbb{R}^{n}\right)$ satisfies (4) and (5) in Proposition 1 with $q_{1}^{+}<n / \gamma, 1 / q_{1}(x)-1 / q_{2}(x)=\gamma / n, \Omega \in L^{s}\left(\mathbb{S}^{n-1}\right)\left(s>q_{2}^{+}\right)$with $1 \leq r^{\prime}<q_{2}^{-}$. Then the commutator $\left[b^{m}, \mu_{\Omega}^{\rho}\right]$ is bounded from $L^{q_{1}(\cdot)}\left(\mathbb{R}^{n}\right)$ to $L^{q_{2}(\cdot)}\left(\mathbb{R}^{n}\right)$.

Lemma 11. [32] Let $p(\cdot) \in \mathcal{P}(\Omega)$ abd $h: \Omega \times \Omega \rightarrow \mathbb{R}$ is a measurable function (with respect to product measure) such that, $y \in \Omega, h(\cdot, y) \in L^{p(\cdot)}(\Omega)$, then we have

$$
\left\|\int_{\Omega} h(\cdot, y) d y\right\|_{L^{p(\cdot)}(\Omega)} \leq C \int_{\Omega}\|h(\cdot, y)\|_{L^{p(\cdot)}(\Omega)} d y .
$$




\section{Main Results}

Theorem 1. Let $0<\rho<n, 0<v \leq 1$. Suppose that $p_{1}(\cdot) \in \mathcal{B}\left(\mathbb{R}^{n}\right), \Omega \in L^{s}\left(\mathbb{S}^{n-1}\right), s>\left(p_{1}^{\prime}\right)_{+}$and $q_{1}(\cdot), q_{2}(\cdot) \in$ $\mathcal{P}\left(\mathbb{R}^{n}\right)$ with $\left(q_{2}\right)_{-} \geq\left(q_{1}\right)_{+}$. If $-n \delta_{1}-v-n / s<\alpha<n \delta_{2}-v-n / s$ with $\delta_{1}, \delta_{2}$ as defined in Lemma 2 , then the operator $\mu_{\Omega}^{\rho}$ is bounded from $\dot{K}_{p_{1}(\cdot)}^{\alpha, q_{1}(\cdot)}\left(\mathbb{R}^{n}\right)$ to $\dot{K}_{p_{1}(\cdot)}^{\alpha, q_{2}(\cdot)}\left(\mathbb{R}^{n}\right)$ and from $\left(K_{p_{1}(\cdot)}^{\alpha, q_{1}(\cdot)}\left(\mathbb{R}^{n}\right)\right)$ to $\left(K_{p_{1}(\cdot)}^{\alpha, q_{2}(\cdot)}\left(\mathbb{R}^{n}\right)\right)$.

Proof. Let $h(x) \in \dot{K}_{p_{1}(\cdot)}^{\alpha, q_{1}(\cdot)}\left(\mathbb{R}^{n}\right)$. Rewrite $h(x)=\sum_{j=-\infty}^{\infty} h(x) \chi_{j}=\sum_{j=-\infty}^{\infty} h_{j}(x)$. From Definition 2, we have

$$
\left\|\mu_{\Omega}^{\rho}(h)\right\|_{\dot{K}_{p_{1}(\cdot)}^{\alpha, q_{2}(\cdot)}\left(\mathbb{R}^{n}\right)}=\inf \left\{\eta>0: \sum_{k=-\infty}^{\infty}\left\|\left(\frac{2^{k \alpha}\left|\mu_{\Omega}^{\rho}(h) \chi_{k}\right|}{\eta}\right)^{q_{2}(\cdot)}\right\|_{L^{\frac{p_{1}(\cdot)}{q_{2}(\cdot)}}} \leq 1\right\} .
$$

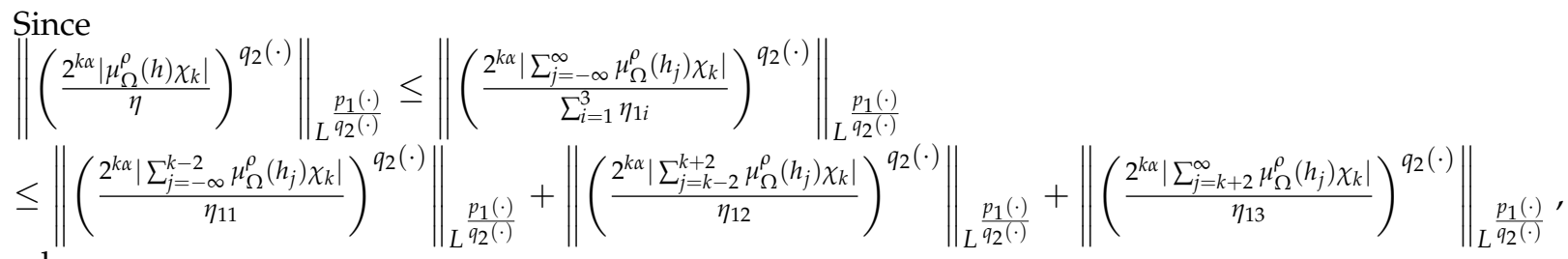

where

$$
\begin{aligned}
& \eta_{11}=\left\|\left\{2^{k \alpha}\left|\sum_{j=-\infty}^{k-2} \mu_{\Omega}^{\rho}\left(h_{j}\right) \chi_{k}\right|\right\}_{k=-\infty}^{\infty}\right\|_{\ell q_{2}(\cdot)\left(L^{p_{1}(\cdot)}\right)}, \\
& \eta_{12}=\left\|\left\{2^{k \alpha}\left|\sum_{j=k-2}^{k+2} \mu_{\Omega}^{\rho}\left(h_{j}\right) \chi_{k}\right|\right\}_{k=-\infty}^{\infty}\right\|_{\ell q_{2}(\cdot)\left(L^{p_{1}(\cdot)}\right)}, \\
& \eta_{13}=\left\|\left\{2^{k \alpha}\left|\sum_{j=k+2}^{\infty} \mu_{\Omega}^{\rho}\left(h_{j}\right) \chi_{k}\right|\right\}_{k=-\infty}^{\infty}\right\|_{\ell^{q_{2}(\cdot)}\left(L^{p_{1}(\cdot)}\right)},
\end{aligned}
$$

and

$$
\eta=\eta_{11}+\eta_{12}+\eta_{13}=\sum_{i=1}^{3} \eta_{1 i}
$$

Thus,

$$
\sum_{k=-\infty}^{\infty}\left\|\left(\frac{2^{k \alpha}\left|\mu_{\Omega}^{\rho}(h) \chi_{k}\right|}{\eta}\right)^{q_{2}(\cdot)}\right\|_{{\frac{1}{p_{2}(\cdot)}}^{q_{2}(\cdot)}} \leq C
$$

Meanwhile,

$$
\left\|\mu_{\Omega}^{\rho}(h)\right\|_{\dot{K}_{p_{1}(\cdot)}^{\alpha, q_{2}(\cdot)}\left(\mathbb{R}^{n}\right)} \leq C \eta=C \sum_{i=1}^{3} \eta_{1 i}
$$

To show Theorem 1 , we only need to estimate $\eta_{11}, \eta_{12}$ and $\eta_{13} \leq C\|h\|_{\dot{K}_{p_{1}(\cdot)}^{\alpha, q_{1}(\cdot)}\left(\mathbb{R}^{n}\right)}$. To do this, denote

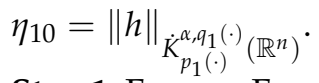

Step 1. For $\eta_{12}$. From Lemma 5 , we get

$$
\begin{aligned}
\sum_{k=-\infty}^{\infty}\left\|\left(\frac{2^{k \alpha}\left|\sum_{j=k-2}^{k+2} \mu_{\Omega}^{\rho}\left(h_{j}\right) \chi_{k}\right|}{\eta_{10}}\right)^{q_{2}(\cdot)}\right\|_{L^{\frac{p_{1}(\cdot)}{q_{2}(\cdot)}}} & \leq \sum_{k=-\infty}^{\infty}\left\|\frac{2^{k \alpha}\left|\sum_{j=k-2}^{k+2} \mu_{\Omega}^{\rho}\left(h_{j}\right) \chi_{k}\right|}{\eta_{10}}\right\|_{L^{p_{1}(\cdot)}}^{\left(q_{2}^{1}\right)_{k}} \\
& \leq \sum_{k=-\infty}^{\infty}\left(\left\|\frac{2^{k \alpha}\left|\sum_{j=k-2}^{k+2} \mu_{\Omega}^{\rho}\left(h_{j}\right) \chi_{k}\right|}{\eta_{10}}\right\|_{L^{p_{1}(\cdot)}}\right)^{\left(q_{2}^{1}\right)_{k}},
\end{aligned}
$$


where

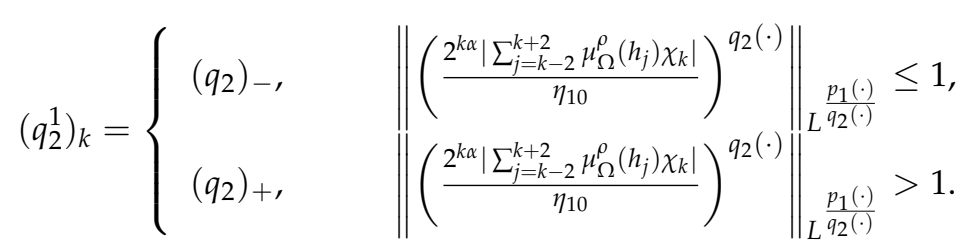

So, by using the Lemma 6, Remark 2 and $h(x) \in \dot{K}_{p_{1}(\cdot)}^{\alpha, q_{1}(\cdot)}\left(\mathbb{R}^{n}\right)$, we have $\left\|\frac{2^{k \alpha}\left|h \chi_{k}\right|}{\eta_{10}}\right\|_{L^{p_{1}(\cdot)}} \leq 1$ and $\sum_{k=-\infty}^{\infty}\left\|\left(\frac{2^{k \alpha}\left|h \chi_{k}\right|}{\eta_{10}}\right)^{q_{1}(\cdot)}\right\|_{L^{\frac{p_{1}(\cdot)}{q_{1}(\cdot)}}} \leq 1$. Hence

$$
\begin{aligned}
& \sum_{k=-\infty}^{\infty}\left\|\left(\frac{2^{k \alpha}\left|\sum_{j=k-2}^{k+2} \mu_{\Omega}^{\rho}\left(h_{j}\right)\left(h_{j}\right) \chi_{k}\right|}{\eta_{10}}\right)^{q_{2}(\cdot)}\right\|_{L_{\frac{p_{1}(\cdot)}{q_{2}(\cdot)}}} \leq C \sum_{k=-\infty}^{\infty}\left(\sum_{j=k-2}^{k+2}\left\|\frac{2^{k \alpha}\left|h_{j}\right|}{\eta_{10}}\right\|_{L^{p_{1}(\cdot)}}\right)^{\left(q_{2}^{1}\right)_{k}} \\
& \leq C \sum_{k=-\infty}^{\infty}\left\|\frac{2^{k \alpha}\left|h \chi_{k}\right|}{\eta_{10}}\right\|_{L^{p_{1}(\cdot)}}^{\left(q_{2}^{1}\right)_{k}} \leq C \sum_{k=-\infty}^{\infty}\left\|\left(\frac{2^{k \alpha}\left|h \chi_{k}\right|}{\eta_{10}}\right)^{q_{1}(\cdot)}\right\| \|_{L^{\frac{p_{1}(\cdot)}{q_{1}(\cdot)}}} \leq C\left\{\sum_{k=-\infty}^{\infty}\left\|\left(\frac{{\left.\frac{(12}{2}\right)_{k}}^{\left(q_{1}\right)+}}{\eta_{10}}\right)^{q_{10} \chi_{k} \mid}\right\|_{L^{\frac{p_{1}(\cdot)}{q_{1}(\cdot)}}}\right\}^{q_{*}} \leq C .
\end{aligned}
$$

Which, together with $\left(p_{1}\right)_{+} \leq\left(p_{2}\right)_{-} \leq\left(q_{2}^{1}\right)_{k}$ and $q_{*}=\min _{k \in N} \frac{\left(q_{2}^{1}\right)_{k}}{\left(q_{1}\right)_{+}}$gives;

$$
\eta_{12} \leq C \eta_{10} \leq C\|h\|_{\dot{K}_{p_{1}(\cdot)}^{\alpha, q_{1}(\cdot)}\left(\mathbb{R}^{n}\right)}
$$

Step 2. Now, let us deal with $\eta_{11}$. Since

$$
\begin{aligned}
\left|\mu_{\Omega}^{\rho}\left(h_{j}\right)(x)\right|: & \left(\int_{0}^{\infty}\left|\int_{|x-y| \leq t} \frac{\Omega(x-y)}{|x-y|^{n-\rho}} h_{j}(y) \mathrm{d} y\right|^{2} \frac{\mathrm{d} t}{t^{2 \rho+1}}\right)^{1 / 2} \\
\leq & \left(\int_{0}^{|x|}\left|\int_{|x-y| \leq t} \frac{\Omega(x-y)}{|x-y|^{n-\rho}} h_{j}(y) \mathrm{d} y\right|^{2} \frac{\mathrm{d} t}{t^{2 \rho+1}}\right)^{1 / 2} \\
& +\left(\int_{|x|}^{\infty}\left|\int_{|x-y| \leq t} \frac{\Omega(x-y)}{|x-y|^{n-\rho}} h_{j}(y) \mathrm{d} y\right|^{2} \frac{\mathrm{d} t}{t^{2 \rho+1}}\right)^{1 / 2} \\
:= & \eta_{11}^{\prime}+\eta_{11}^{\prime \prime} .
\end{aligned}
$$

Now we estimate $\eta_{11}^{\prime}$ and $\eta_{11}^{\prime \prime}$. For $\eta_{11}^{\prime}$, note that $x \in A_{k}, y \in A_{j}$ and $j \leq k-2$. Since $|x-y| \sim|x|$ so by virtue of the Mean Value Theorem, we have

$$
\left|\frac{1}{|x-y|^{2 \rho}}-\frac{1}{|x|^{2 \rho}}\right| \leq C \frac{|y|}{|x-y|^{2 \rho+1}}
$$

Substituting the inequality (9) into $\eta_{11}^{\prime}$ and by virtue of Minkowski's inequality, we deduced that

$$
\begin{aligned}
\eta_{11}^{\prime} & \leq C \int_{\mathbb{R}^{n}} \frac{|\Omega(x-y)|}{|x-y|^{n-\rho}}\left|h_{j}(y)\right|\left(\int_{|x-y|}^{|x|} \frac{\mathrm{d} t}{t^{2 \rho+1}}\right)^{1 / 2} \mathrm{~d} y \leq C \int_{\mathbb{R}^{n}} \frac{|\Omega(x-y)|}{|x-y|^{n-\rho}}\left|h_{j}(y)\right|\left|\frac{1}{|x-y|^{2 \rho}}-\frac{1}{|x|^{2 \rho}}\right|^{1 / 2} \mathrm{~d} y \\
& \leq C \int_{\mathbb{R}^{n}} \frac{|\Omega(x-y)|}{|x-y|^{n-\rho}}\left|h_{j}(y)\right| \frac{|y|^{1 / 2}}{|x-y|^{\rho+1 / 2}} \mathrm{~d} y \leq C \frac{2^{j / 2}}{|x|^{n+1 / 2}} \int_{A_{j}}|\Omega(x-y)|\left|h_{j}(y)\right| \mathrm{d} y \\
& \leq C 2^{j / 2} 2^{-k(n+1 / 2)} \int_{A_{j}}|\Omega(x-y)|\left|h_{j}(y)\right| \mathrm{d} y \leq C 2^{(j-k) / 2} 2^{-n k} \int_{A_{j}}|\Omega(x-y)|\left|h_{j}(y)\right| \mathrm{d} y .
\end{aligned}
$$

Similarly, we obtain 


$$
\begin{aligned}
\eta_{11}^{\prime \prime} & \leq C \int_{\mathbb{R}^{n}} \frac{|\Omega(x-y)|}{|x-y|^{n-\rho}}\left|h_{j}(y)\right|\left(\int_{|x|}^{\infty} \frac{\mathrm{d} t}{t^{2 \rho+1}}\right)^{1 / 2} \mathrm{~d} y \leq C \int_{\mathbb{R}^{n}} \frac{|\Omega(x-y)|}{|x-y|^{n-\rho}}\left|h_{j}(y)\right|\left(\frac{1}{|x|^{2 \rho}}\right)^{1 / 2} \mathrm{~d} y \\
& \leq C \int_{\mathbb{R}^{n}} \frac{|\Omega(x-y)|}{|x-y|^{n}}\left|h_{j}(y)\right| \mathrm{d} y \leq C 2^{-n k} \int_{A_{j}}|\Omega(x-y)|\left|h_{j}(y)\right| \mathrm{d} y .
\end{aligned}
$$

Combining the inequality (11) with Lemma 1, we get

$$
\left|\mu_{\Omega}^{\rho}\left(h_{j}\right)(x)\right| \leq C 2^{-n k} \int_{A_{j}}|\Omega(x-y)|\left|h_{j}(y)\right| \mathrm{d} y \leq C 2^{-n k}\left\|(\Omega(x-\cdot)) \cdot \chi_{B_{j}}\right\|_{L^{p_{1}^{\prime}(\cdot)}}\left\|h_{j}\right\|_{L^{p_{1}(\cdot)}} .
$$

Now, consider $\tilde{p}_{1}^{\prime}(\cdot)>1$ and $1 / p_{1}^{\prime}(x)=1 / \tilde{p}_{1}^{\prime}(x)+1 /$ s. Since $s>\left(p_{1}^{\prime}\right)_{+}$, so by virtue of Lemma 1 and Lemma 8 , we get

$$
\begin{aligned}
& \left\|(\Omega(x-\cdot)) \cdot \chi_{B_{j}}\right\|_{L^{p_{1}^{\prime}(\cdot)}} \leq\|\Omega(x-\cdot)\|\left\|_{L^{s}}\right\| \chi_{B_{j}}\left\|_{L^{\tilde{p}^{\prime}(\cdot)}} \leq 2^{-j v}\left(\int_{A_{j}}|y|^{s v}|\Omega(x-y)|^{s} \mathrm{~d} y\right)^{1 / s}\right\| \chi_{B_{j}} \|_{L^{\tilde{p}_{1}^{\prime}(\cdot)}} \\
& \leq 2^{-j v} 2^{k(v+n / s)}\|\Omega\|_{L^{s}\left(\mathbb{S}^{n-1}\right)}\left\|\chi_{B_{j}}\right\|_{L^{\tilde{p}_{1}^{\prime}(\cdot)}} \leq 2^{-j v} 2^{k(v+n / s)}\|\Omega\|_{L^{s}\left(\mathbb{S}^{n-1}\right)}\left\|\chi_{B_{j}}\right\|_{L^{p_{1}^{\prime}(\cdot)}} /\left|B_{j}\right|^{1 / s} \\
& \leq 2^{(k-j)(v+n / s)}\|\Omega\|_{L^{s}\left(\mathbb{S}^{n-1}\right)}\left\|\chi_{B_{j}}\right\|_{L^{p_{1}^{\prime}(\cdot)}} .
\end{aligned}
$$

By using (12), (13), Lemmas 1, 2, 3, 5 and $\left\|\frac{2^{j \alpha}\left|h \chi_{j}\right|}{\eta_{10}}\right\|_{L^{p_{1}(\cdot) q_{1}}} \leq 1$, we get

$$
\begin{aligned}
& \sum_{k=-\infty}^{\infty}\left\|\left(\frac{2^{k \alpha}\left|\sum_{j=-\infty}^{k-2} \mu_{\Omega}^{\rho}\left(h_{j}\right) \chi_{k}\right|}{\eta_{10}}\right)^{q_{2}(\cdot)}\right\|_{L^{\frac{p_{1}(\cdot)}{q_{2}(\cdot)}}} \leq C \sum_{k=-\infty}^{\infty}\left(\left\|\frac{2^{k \alpha}\left|\sum_{j=-}^{\infty} \mu_{\Omega}^{\rho}\left(h_{j}\right) \chi_{k}\right|}{\eta_{10}}\right\|_{L^{p_{1}(\cdot)}\left(\mathbb{R}^{n}\right)}\right)^{\left(q_{2}^{2}\right)_{k}} \\
& \leq C \sum_{k=-\infty}^{\infty}\left(2^{k \alpha} \sum_{j=-\infty}^{k-2} 2^{-k n} 2^{(k-j)(v+n / s)}\left\|\frac{h_{j}}{\eta_{10}}\right\|_{L^{p_{1}(\cdot)\left(\mathbb{R}^{n}\right)}}\left\|\chi_{B_{k}}\right\|_{L^{p_{1}(\cdot)}\left(\mathbb{R}^{n}\right)}\left\|\chi_{B_{j}}\right\|_{L^{p_{1}^{\prime}(\cdot)}\left(\mathbb{R}^{n}\right)}\right)^{\left(q_{2}^{2}\right)_{k}} \\
& \leq C \sum_{k=-\infty}^{\infty}\left(2^{k \alpha} \sum_{j=-\infty}^{k-2} 2^{(k-j)(v+n / s)}\left\|\frac{h \chi_{j}}{\eta_{10}}\right\|_{L^{p_{1}(\cdot)}\left(\mathbb{R}^{n}\right)} \frac{\left\|\chi_{B_{j}}\right\|_{L^{p_{1}^{\prime}(\cdot)}\left(\mathbb{R}^{n}\right)}}{\left\|\chi_{B_{k}}\right\|_{L^{p_{1}^{\prime}(\cdot)}\left(\mathbb{R}^{n}\right)}}\right)^{\left(q_{2}^{2}\right)_{k}} \\
& \leq C \sum_{k=-\infty}^{\infty}\left(2^{k \alpha} \sum_{j=-\infty}^{k-2} 2^{(k-j)(v+n / s)} 2^{-j \alpha}\left\|\frac{\left|2^{j \alpha} h \chi_{j}\right|}{\eta_{10}}\right\|_{L^{p_{1}(\cdot)}\left(\mathbb{R}^{n}\right)} \frac{\left\|\chi_{B_{j}}\right\|_{L^{p_{1}^{\prime}(\cdot)}\left(\mathbb{R}^{n}\right)}}{\left\|\chi_{B_{k}}\right\|_{L^{p_{1}^{\prime}(\cdot)}\left(\mathbb{R}^{n}\right)}}\right)^{\left(q_{2}^{2}\right)_{k}} \\
& \leq C \sum_{k=-\infty}^{\infty}\left\{\sum_{j=-\infty}^{k-2} 2^{(k-j)\left(\alpha+v+n / s-n \delta_{2}\right)}\left\|\left(\frac{\left|2^{j \alpha} h \chi_{j}\right|}{\eta_{10}}\right)^{q_{1}(\cdot)}\right\|_{L^{p_{1}(\cdot) q_{1}(\cdot)}\left(\mathbb{R}^{n}\right)}^{\frac{1}{\left(q_{1}\right)+}}\right\}^{\left(q_{2}^{2}\right)_{k}},
\end{aligned}
$$

where

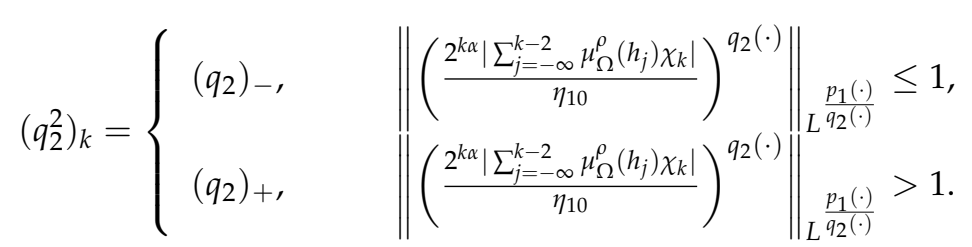

Which, together with $\left(q_{1}\right)_{+}<1$ and $\left(p_{1}\right)_{+} \leq\left(p_{2}\right)_{-} \leq\left(q_{2}^{2}\right)_{k}$ gives;

$$
\begin{aligned}
\sum_{k=-\infty}^{\infty}\left\|\left(\frac{2^{k \alpha}\left|\sum_{j=-\infty}^{k-2} \mu_{\Omega}^{\rho}\left(h_{j}\right) \chi_{k}\right|}{\eta_{10}}\right)^{q_{2}(\cdot)}\right\|_{L^{\frac{p_{1}(\cdot)}{q_{2}(\cdot)}}} \leq C\left\{\sum_{j=-\infty}^{\infty}\left\|\left(\frac{\left|2^{j \alpha} h \chi_{j}\right|}{\eta_{10}}\right)^{q_{1}(\cdot)}\right\|_{L^{p_{1}(\cdot) q_{1}(\cdot)}} \sum_{k=j+2}^{\infty} 2^{(k-j)\left(\alpha+v+n / s-n \delta_{2}\right)}\right\}^{q_{*}} \\
\leq C
\end{aligned}
$$

where $q_{*}=\min _{k \in \mathbb{N}} \frac{\left(q_{2}^{1}\right)_{k}}{\left(q_{1}\right)_{+}}$. 
Since $\alpha<n \delta_{2}-(v+n / s)$, so if $\left(q_{1}\right)_{+} \geq 1$ and $\left(q_{2}^{2}\right)_{k} \geq\left(q_{2}\right)_{-} \geq\left(q_{1}\right)_{+} \geq 1$ then by using Remark 2and applying the generalized Hölder's inequality, we get

$$
\begin{aligned}
& \sum_{k=-\infty}^{\infty}\left\|\left(\frac{2^{k \alpha}\left|\sum_{j=-\infty}^{k-2} \mu_{\Omega}^{\rho}\left(h_{j}\right) \chi_{k}\right|}{\eta_{10}}\right)^{q_{2}(\cdot)}\right\|_{L^{\frac{p_{1}(\cdot)}{q_{2}(\cdot)}}} \leq C \sum_{k=-\infty}^{\infty}\left\{\sum_{j=-\infty}^{k-2} 2^{(k-j)\left(\alpha+v+n / s-n \delta_{2}\right)\left(q_{1}\right)_{+} / 2}\left\|\left(\frac{\left|2^{j \alpha} h \chi_{j}\right|}{\eta_{10}}\right)^{q_{1}(\cdot)}\right\|_{L^{p_{1}(\cdot) q_{1}(\cdot)}}\right\}^{\frac{\left(q_{2}^{2}\right)_{k}}{\left(q_{1}\right)+}} \\
& \left.\quad \times\left(\sum_{j=-\infty}^{k-2} 2^{(k-j)\left(\alpha+v+n / s-n \delta_{2}\right)\left(\left(q_{1}\right)_{+}\right)^{\prime} / 2}\right)^{\frac{\left(q_{2}^{2}\right)_{k}}{\left(\left(q_{1}\right)+\right)^{\prime}}}\right)^{\leq C}\left\{\sum_{j=-\infty}^{\infty}\left\|\left(\frac{\left|2^{j \alpha} h \chi_{j}\right|}{\eta_{10}}\right)^{q_{1}(\cdot)}\right\| \sum_{L^{p_{1}(\cdot) q_{1}(\cdot)}} \sum_{k=j+2}^{\infty} 2^{(k-j)\left(\alpha+v+n / s-n \delta_{2}\right)\left(q_{1}\right)_{+} / 2}\right\}^{q_{*}} \\
& \leq C,
\end{aligned}
$$

where $q_{*}=\min _{k \in \mathbb{N}} \frac{\left(q_{2}^{2}\right)_{k}}{\left(q_{1}\right)_{+}}$. Hence we have

$$
\eta_{11} \leq C \eta_{10} \leq C\|h\|_{\dot{K}_{p_{1}(\cdot)}^{\alpha, q_{1}(\cdot)}\left(\mathbb{R}^{n}\right)} .
$$

Step 3. Finally, we estimate $\eta_{13}$. For each $x \in A_{j}$ and $j \geq k+2$, we have

$$
\begin{aligned}
\left|\mu_{\Omega}^{\rho}\left(h_{j}\right)(x)\right|:= & \left(\int_{0}^{\infty}\left|\int_{|x-y| \leq t} \frac{\Omega(x-y)}{|x-y|^{n-\rho}} h_{j}(y) \mathrm{d} y\right|^{2} \frac{\mathrm{d} t}{t^{2 \rho+1}}\right)^{1 / 2} \\
\leq & \left(\int_{0}^{|y|}\left|\int_{|x-y| \leq t} \frac{\Omega(x-y)}{|x-y|^{n-\rho}} h_{j}(y) \mathrm{d} y\right|^{2} \frac{\mathrm{d} t}{t^{2 \rho+1}}\right)^{1 / 2} \\
& +\left(\int_{|y|}^{\infty}\left|\int_{|x-y| \leq t} \frac{\Omega(x-y)}{|x-y|^{n-\rho}} h_{j}(y) \mathrm{d} y\right|^{2} \frac{\mathrm{d} t}{t^{2 \rho+1}}\right)^{1 / 2} \\
:= & \eta_{13}^{\prime}+\eta_{13}^{\prime \prime} .
\end{aligned}
$$

The estimates of $\eta_{13}^{\prime}$ and $\eta_{13}^{\prime \prime}$ can be obtained similarly as that of $\eta_{11}^{\prime}$ and $\eta_{11}^{\prime \prime}$ in Step 2 and we get

$$
\eta_{13}^{\prime} \leq C 2^{(j-k) / 2} 2^{-j n} \int_{A_{j}}|\Omega(x-y)|\left|h_{j}(y)\right| \mathrm{d} y,
$$

and

$$
\eta_{13}^{\prime \prime} \leq C 2^{-j n} \int_{A_{j}}|\Omega(x-y)|\left|h_{j}(y)\right| \mathrm{d} y
$$

Thus, we have

$$
\left|\mu_{\Omega}^{\rho}\left(h_{j}\right)(x)\right| \leq C 2^{-j n} \int_{A_{j}}|\Omega(x-y)|\left|h_{j}(y)\right| \mathrm{d} y \leq C 2^{-j n}\left\|(\Omega(x-\cdot)) \cdot \chi_{B_{j}}\right\|_{L^{p^{\prime}(\cdot)}}\left\|h_{j}\right\|_{L^{p(\cdot)}} .
$$

Substituting (13) into (20), together with Lemmas 1, 2, 3, 5 and $\left\|\frac{2^{j \alpha}\left|h \chi_{j}\right|}{\eta_{10}}\right\|_{L^{p_{1}(\cdot) q_{1}(\cdot)}} \leq 1$, we get 


$$
\begin{aligned}
& \sum_{k=-\infty}^{\infty}\left\|\left(\frac{2^{k \alpha}\left|\sum_{j=k+2}^{\infty} \mu_{\Omega}^{\rho}\left(h_{j}\right) \chi_{k}\right|}{\eta_{10}}\right)^{q_{2}(\cdot)}\right\|_{L^{\frac{p_{1}(\cdot)}{q_{2}(\cdot)}}} \leq C \sum_{k=-\infty}^{\infty}\left\|\frac{2^{k \alpha}\left|\sum_{j=k+2}^{\infty} \mu_{\Omega}^{\rho}\left(h_{j}\right) \chi_{k}\right|}{\eta_{10}}\right\|_{L^{p_{1}(\cdot)}}^{\left(\eta_{2}^{3}\right)_{k}} \\
& \leq C \sum_{k=-\infty}^{\infty}\left(2^{k \alpha} \sum_{j=k+2}^{\infty} 2^{-j n} 2^{(k-j)(v+n / s)} \times\left\|\frac{h_{j}}{\eta_{10}}\right\|_{L^{p_{1}(\cdot)\left(\mathbb{R}^{n}\right)}}\left\|\chi_{B_{k}}\right\|_{L^{p_{1}(\cdot)\left(\mathbb{R}^{n}\right)}}\left\|\chi_{B_{j}}\right\|_{L^{p_{1}^{\prime}(\cdot)}\left(\mathbb{R}^{n}\right)}\right)^{\left(q_{2}^{3}\right)_{k}} \\
& \leq C \sum_{k=-\infty}^{\infty}\left(2^{k \alpha} \sum_{j=k+2}^{\infty} 2^{-j n} 2^{(k-j)(v+n / s)}\left\|\frac{h \chi_{j}}{\eta_{10}}\right\|_{L^{p_{1}(\cdot)\left(\mathbb{R}^{n}\right)}}\left|B_{j}\right| \frac{\left\|\chi_{B_{j}}\right\|_{L_{1}^{p_{1}^{\prime}(\cdot)}\left(\mathbb{R}^{n}\right)}}{\left\|\chi_{B_{j}}\right\|_{L^{p_{1}(\cdot)}\left(\mathbb{R}^{n}\right)}}\right)^{\left(q_{2}^{3}\right)_{k}} \\
& \leq C \sum_{k=-\infty}^{\infty}\left(2^{k \alpha} \sum_{j=k+2}^{\infty} 2^{(k-j)(v+n / s)}\left\|\frac{h \chi_{j}}{\eta_{10}}\right\|_{L^{p_{1}(\cdot)}\left(\mathbb{R}^{n}\right)} \frac{\left\|\chi_{B_{j}}\right\|_{L^{p_{1}^{\prime}(\cdot)}\left(\mathbb{R}^{n}\right)}}{\left\|\chi_{B_{j}}\right\|_{L^{p_{1}(\cdot)}\left(\mathbb{R}^{n}\right)}}\right)^{\left(q_{2}^{3}\right)_{k}} \\
& \leq C \sum_{k=-\infty}^{\infty}\left(2^{k \alpha} \sum_{j=k+2}^{\infty} 2^{(k-j)(v+n / s)} 2^{-j \alpha}\left\|\frac{\left|2^{j \alpha} h \chi_{j}\right|}{\eta_{10}}\right\|_{L^{p_{1}(\cdot)}\left(\mathbb{R}^{n}\right)} \frac{\left\|\chi_{B_{k}}\right\|_{L^{p_{1}(\cdot)}\left(\mathbb{R}^{n}\right)}}{\left\|\chi_{j_{k}}\right\|_{L^{p_{1}(\cdot)}\left(\mathbb{R}^{n}\right)}}\right)^{\left(q_{2}^{3}\right)_{k}} \\
& \leq C \sum_{k=-\infty}^{\infty}\left\{\sum_{j=k+2}^{\infty} 2^{(k-j)\left(\alpha+v+n / s+n \delta_{12}\right)}\left\|\left(\frac{\left|2^{j \alpha} h \chi_{j}\right|}{\eta_{10}}\right)^{q_{1}(\cdot)}\right\|_{L^{p_{1}(\cdot) q_{1}(\cdot)}\left(\mathbb{R}^{n}\right)}^{\frac{1}{\left(q_{1}\right)+}}\right\}^{\left(q_{2}^{3}\right)_{k}},
\end{aligned}
$$

where

$$
\left(q_{2}^{3}\right)_{k}=\left\{\begin{array}{l|l}
\left(q_{2}\right)_{-}, & \left\|\left(\frac{2^{k \alpha}\left|\sum_{j=k+2}^{\infty} \mu_{\Omega}^{\rho}\left(h_{j}\right) \chi_{k}\right|}{\eta_{10}}\right)^{q_{2}(\cdot)}\right\| \\
\left(q_{2}\right)_{+}, & \left\|\left(\frac{2^{k \alpha}\left|\sum_{j=k+2}^{\infty} \mu_{\Omega}^{\rho}\left(h_{j}\right) \chi_{k}\right|}{\eta_{10}}\right)^{q_{2}(\cdot)}\right\|_{L^{\frac{p_{1}(\cdot)}{q_{2}(\cdot)}} \leq 1,}^{\frac{p_{1}(\cdot)}{q_{2}(\cdot)}}>1 .
\end{array}\right.
$$

From above and by an argument similar to that of Step 2, we conclude

$$
\eta_{13} \leq C \eta_{10} \leq C\|h\|_{\dot{K}_{p_{1}(\cdot)}^{\alpha, q_{1}(\cdot)}\left(\mathbb{R}^{n}\right)} .
$$

The proof is completed.

Theorem 2. Suppose $b \in \mathrm{BMO}\left(\mathbb{R}^{n}\right), m \in \mathbb{N}, 0<\rho<n, 0<v \leq 1$. Further suppose that $p_{1}(\cdot) \in \mathcal{B}\left(\mathbb{R}^{n}\right), \Omega \in$ $L^{s}\left(\mathbb{S}^{n-1}\right), s>\left(p_{1}^{\prime}\right)_{+}$and $q_{1}(\cdot), q_{2}(\cdot) \in \mathcal{P}\left(\mathbb{R}^{n}\right)$ with $\left(q_{2}\right)_{-} \geq\left(q_{1}\right)_{+}$. If $-n \delta_{1}-v-n / s<\alpha<n \delta_{2}-v-$ $n / s$ with $\delta_{1}, \delta_{2}$ as defined in Lemma 2. Then the operator $\left[b^{m}, \mu_{\Omega}^{\rho}\right]$ is bounded from $\dot{K}_{p_{1}(\cdot)}^{\alpha, q_{1}(\cdot)}\left(\mathbb{R}^{n}\right)$ to $\dot{K}_{p_{1}(\cdot)}^{\alpha, q_{2}(\cdot)}\left(\mathbb{R}^{n}\right)$ and $\left(K_{p_{1}(\cdot)}^{\alpha, q_{1}(\cdot)}\left(\mathbb{R}^{n}\right)\right)$ to $\left(K_{p_{1}(\cdot)}^{\alpha, q_{2}(\cdot)}\left(\mathbb{R}^{n}\right)\right)$.

Proof. Let $h(x) \in \dot{K}_{p_{1}(\cdot)}^{\alpha, q_{1}(\cdot)}\left(\mathbb{R}^{n}\right), b \in \mathrm{BMO}\left(\mathbb{R}^{n}\right)$. We may write $h(x)=\sum_{j=-\infty}^{\infty} h(x) \chi_{j}=\sum_{j=-\infty}^{\infty} h_{j}(x)$. By definition of $\dot{K}_{p(\cdot)}^{\alpha, q(\cdot)}\left(\mathbb{R}^{n}\right)$, we have

$$
\left\|\left[b^{m}, \mu_{\Omega}^{\rho}\right](h)\right\|_{\dot{K}_{p_{1}(\cdot)}^{\alpha, q_{2}(\cdot)}\left(\mathbb{R}^{n}\right)}=\inf \left\{\eta>0: \sum_{k=-\infty}^{\infty}\left\|\left(\frac{2^{k \alpha}\left|\left[b^{m}, \mu_{\Omega}^{\rho}\right](h) \chi_{k}\right|}{\eta}\right)^{q_{2}(\cdot)}\right\|_{L^{\frac{p_{1}(\cdot)}{q_{2}(\cdot)}}} \leq 1\right\} .
$$

Since

$$
\left\|\left(\frac{2^{k \alpha}\left|\left[b^{m}, \mu_{\Omega}^{\rho}\right](h) \chi_{k}\right|}{\eta}\right)^{q_{2}(\cdot)}\right\|_{L^{\frac{p_{1}(\cdot)}{q_{2}(\cdot)}}} \leq\left\|\left(\frac{2^{k \alpha}\left|\sum_{j=-\infty}^{k-2}\left[b^{m}, \mu_{\Omega}^{\rho}\right]\left(h_{j}\right) \chi_{k}\right|}{\sum_{i=1}^{3} \eta_{2 i}}\right)^{q_{2}(\cdot)}\right\|_{L^{\frac{p_{1}(\cdot)}{q_{2}(\cdot)}}}
$$




$$
\begin{aligned}
& \leq\left\|\left(\frac{2^{k \alpha}\left|\sum_{j=-\infty}^{\infty}\left[b^{m}, \mu_{\Omega}^{\rho}\right]\left(h_{j}\right) \chi_{k}\right|}{\eta_{21}}\right)^{q_{2}(\cdot)}\right\|_{L^{\frac{p_{1}(\cdot)}{q_{2}(\cdot)}}}+\left\|\left(\frac{2^{k \alpha}\left|\sum_{j=k-2}^{k+2}\left[b^{m}, \mu_{\Omega}^{\rho}\right]\left(h_{j}\right) \chi_{k}\right|}{\eta_{22}}\right)^{q_{2}(\cdot)}\right\|_{L^{\frac{p_{1}(\cdot)}{q_{2}(\cdot)}}} \\
& +\left\|\left(\frac{2^{k \alpha}\left|\sum_{j=k+2}^{\infty}\left[b^{m}, \mu_{\Omega}^{\rho}\right]\left(h_{j}\right) \chi_{k}\right|}{\eta_{23}}\right)^{q_{2}(\cdot)}\right\|_{L_{\frac{p_{1}(\cdot)}{q_{2}(\cdot)}}}
\end{aligned}
$$

Let

$$
\begin{aligned}
& \eta_{21}=\left\|\left\{2^{k \alpha}\left|\sum_{j=-\infty}^{k-2}\left[b^{m}, \mu_{\Omega}^{\rho}\right]\left(h_{j}\right) \chi_{k}\right|\right\}_{k=-\infty}^{\infty}\right\|_{\ell q_{2}(\cdot)\left(L^{p_{1}(\cdot)}\right)}, \\
& \eta_{22}=\left\|\left\{2^{k \alpha}\left|\sum_{j=k-2}^{k+2}\left[b^{m}, \mu_{\Omega}^{\rho}\right]\left(h_{j}\right) \chi_{k}\right|\right\}_{k=-\infty}^{\infty}\right\|_{\ell^{q_{2}(\cdot)}\left(L^{p_{1}(\cdot)}\right)}, \\
& \eta_{23}=\left\|\left\{2^{k \alpha}\left|\sum_{j=k+2}^{\infty}\left[b^{m}, \mu_{\Omega}^{\rho}\right]\left(h_{j}\right) \chi_{k}\right|\right\}_{k=-\infty}^{\infty}\right\|_{\ell^{q_{2}(\cdot)}\left(L^{p_{1}(\cdot)}\right)},
\end{aligned}
$$

where we put

$$
\eta=\eta_{21}+\eta_{22}+\eta_{23}=\sum_{i=1}^{3} \eta_{2 i}
$$

Hence,

$$
\sum_{k=-\infty}^{\infty}\left\|\left(\frac{2^{k \alpha}\left|\left[b^{m}, \mu_{\Omega}^{\rho}\right](h) \chi_{k}\right|}{\eta}\right)^{q_{2}(\cdot)}\right\|_{L^{\frac{p_{1}(\cdot)}{q_{2}(\cdot)}}} \leq C .
$$

So, it follows that

$$
\left\|\left[b^{m}, \mu_{\Omega}^{\rho}\right](h)\right\|_{\dot{K}_{p_{1}(\cdot)}^{\alpha, q_{2}(\cdot)}\left(\mathbb{R}^{n}\right)} \leq C \eta=C \sum_{i=1}^{3} \eta_{1 i} .
$$

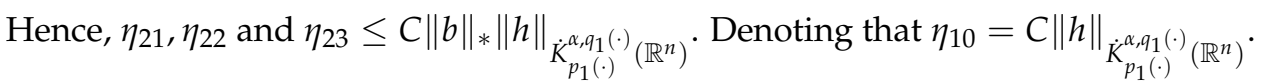

Step 1. We estimate $\eta_{22}$. The proof of Theorem 2 is the same to that of Theorem 1 and we use the similar notation as in the proof $\eta_{12}$ of Theorem 1 . By Lemma 5 and $\left(L^{p(\cdot)}\left(\mathbb{R}^{n}\right), L^{p(\cdot)}\left(\mathbb{R}^{n}\right)\right)$-boundedness of the operators $\left[b^{m}, \mu_{\Omega}^{\rho}\right]$, we directly arrive at

$$
\sum_{k=-\infty}^{\infty}\left\|\left(\frac{2^{k \alpha}\left|\sum_{j=k-2}^{k+2}\left[b^{m}, \mu_{\Omega}^{\rho}\right]\left(h_{j}\right) \chi_{k}\right|}{\eta_{10}\|b\|_{*}}\right)^{q_{2}(\cdot)}\right\|_{L^{\frac{p_{1}(\cdot)}{q_{2}(\cdot)}}} \leq C,
$$

which, implies that

$$
\eta_{21} \leq C \eta_{10}\|b\|_{*} \leq C\|b\|_{*}\|h\|_{\dot{K}_{p_{1}(\cdot)}^{\alpha, q_{1}(\cdot)}\left(\mathbb{R}^{n}\right)} .
$$

Step 2. Next we estimate $\eta_{21}$. Since

$$
\begin{aligned}
\left|\left[b^{m}, \mu_{\Omega}^{\rho}\right]\left(h_{j}\right)(x)\right|:= & \left(\int_{0}^{\infty}\left|\int_{|x-y| \leq t} \frac{\Omega(x-y)}{|x-y|^{n-\rho}}[b(x)-b(y)]^{m} h_{j}(y) \mathrm{d} y\right|^{2} \frac{\mathrm{d} t}{t^{2 \rho+1}}\right)^{1 / 2} \\
\leq & \left(\int_{0}^{|x|}\left|\int_{|x-y| \leq t} \frac{\Omega(x-y)}{|x-y|^{n-\rho}}[b(x)-b(y)]^{m} h_{j}(y) \mathrm{d} y\right|^{2} \frac{\mathrm{d} t}{t^{2 \rho+1}}\right)^{1 / 2} \\
& +\left(\int_{|x|}^{\infty}\left|\int_{|x-y| \leq t} \frac{\Omega(x-y)}{|x-y|^{n-\rho}}[b(x)-b(y)]^{m} h_{j}(y) \mathrm{d} y\right|^{2} \frac{\mathrm{d} t}{t^{2 \rho+1}}\right)^{1 / 2} \\
:= & { }^{\prime 2}{ }^{\prime \prime}{ }^{\prime 2} .
\end{aligned}
$$


Observe that $|x-y| \approx|x|$ for each $x \in A_{k}, y \in A_{j}$ and $j \leq k-2$. From (9) and applying the Minkowski's and the generalized Hölder's inequality, we get

$$
\begin{aligned}
& { }_{22} \leq C \int_{\mathbb{R}^{n}} \frac{|\Omega(x-y)|}{|x-y|^{n-\rho}}[b(x)-b(y)]^{m}\left|h_{j}(y)\right|\left(\int_{|x-y|}^{|x|} \frac{\mathrm{d} t}{t^{2 \rho+1}}\right)^{1 / 2} \mathrm{~d} y \\
& \leq C \int_{\mathbb{R}^{n}} \frac{|\Omega(x-y)|}{|x-y|^{n-\rho}}[b(x)-b(y)]^{m}\left|h_{j}(y)\right| \frac{1}{|x-y|^{2 \rho}}-\left.\frac{1}{|x|^{2 \rho}}\right|^{1 / 2} \mathrm{~d} y \\
& \leq C \int_{\mathbb{R}^{n}} \frac{|\Omega(x-y)|}{|x-y|^{n-\rho}}[b(x)-b(y)]^{m}\left|h_{j}(y)\right| \frac{|y|^{1 / 2}}{|x-y|^{\rho+1 / 2}} \mathrm{~d} y \\
& \leq C \frac{2^{j / 2}}{|x|^{n+1 / 2}}\left\{\left[b(x)-b_{B_{j}}\right]^{m} \int_{A_{j}}|\Omega(x-y)|\left|h_{j}(y)\right| \mathrm{d} y+\int_{A_{j}}|\Omega(x-y)|\left[b_{B_{j}}-b(y)\right]^{m}\left|h_{j}(y)\right| \mathrm{d} y\right\} \\
& \leq C 2^{j / 2} 2^{-k(n+1 / 2)}\left\{\left[b(x)-b_{B_{j}}\right]^{m}\left\|(\Omega(x-\cdot)) \cdot \chi_{B_{j}}\right\|_{L^{p_{1}^{\prime}(\cdot)}}\left\|h_{j}\right\|_{L^{p_{1}(\cdot)}}\right. \\
& \left.+\left\|\Omega(x-\cdot)\left(b_{B_{j}}-b(\cdot)\right)^{m}\left(h_{j}\right) \cdot \chi_{B_{j}}\right\|_{L^{p_{1}^{\prime}(\cdot)}}\left\|h_{j}\right\|_{L^{p_{1}(\cdot)}}\right\} .
\end{aligned}
$$

Similarly, we consider " 22

$$
\begin{aligned}
{ }_{22} \leq & C \int_{\mathbb{R}^{n}} \frac{|\Omega(x-y)|}{|x-y|^{n-\rho}}\left[b(x)-b_{B_{j}}\right]^{m}\left|h_{j}(y)\right|\left(\int_{|x|}^{\infty} \frac{\mathrm{d} t}{t^{2 \rho+1}}\right)^{1 / 2} \mathrm{~d} y \\
\leq & C \int_{\mathbb{R}^{n}} \frac{|\Omega(x-y)|}{|x-y|^{n-\rho}}\left[b(x)-b_{B_{j}}\right]^{m}\left|h_{j}(y)\right|\left(\frac{1}{|x|^{2 \rho}}\right)^{1 / 2} \mathrm{~d} y \\
\leq & C 2^{-n k}\left\{\left[b(x)-b_{B_{j}}\right]^{m} \int_{A_{j}}|\Omega(x-y)|\left|h_{j}(y)\right| \mathrm{d} y+\int_{A_{j}}|\Omega(x-y)|\left[b_{B_{j}}-b(y)\right]^{m}\left|h_{j}(y)\right| \mathrm{d} y\right\} \\
\leq & C 2^{-n k}\left\{\left[b(x)-b_{B_{j}}\right]^{m}\left\|(\Omega(x-\cdot)) \cdot \chi_{j}(\cdot)\right\|_{L^{p_{1}^{\prime}(\cdot)}\left\|h_{j}\right\|_{L^{p_{1}(\cdot)}}}\right. \\
& \left.\quad+\left\|\Omega(x-\cdot)\left(b_{B_{j}}-b(\cdot)\right)^{m}\left(h_{j}\right) \cdot \chi_{j}(\cdot)\right\|_{L^{p_{1}^{\prime}(\cdot)}}\left\|h_{j}\right\|_{L^{p_{1}(\cdot)}}\right\} .
\end{aligned}
$$

Therefore,

$$
\begin{aligned}
\left|\left[b^{m}, \mu_{\Omega}^{\rho}\right]\left(h_{j}\right)(x)\right| \leq & C 2^{-n k}\left\{\left[b(x)-b_{B_{j}}\right]^{m}\left\|(\Omega(x-\cdot)) \cdot \chi_{j}(\cdot)\right\|_{L^{\left.p_{1}^{(} \cdot\right)}}\left\|h_{j}\right\|_{L^{p(\cdot)}}\right. \\
& +\left\|\Omega(x-\cdot)\left(b_{B_{j}}-b(\cdot)\right)^{m} \cdot \chi_{j}(\cdot)\right\|_{L^{p_{1}^{\prime}(\cdot)}}\left\|h_{j}\right\|_{\left.L^{p_{1}(\cdot)}\right\} .}
\end{aligned}
$$

By (13) and Lemmas 6 and 7, we get

$$
\begin{aligned}
& \left\|\Omega(x-\cdot)\left(b_{B_{j}}-b(\cdot)\right)^{m} \cdot \chi_{j}(\cdot)\right\|_{L^{p_{1}^{(}(\cdot)}} \leq\left\|\Omega(x-\cdot) \cdot \chi_{j}(\cdot)\right\|_{L^{s}}\left\|\left(b_{B_{j}}-b(\cdot)\right)^{m} \cdot \chi_{j}(\cdot)\right\|_{L^{\tilde{p}_{1}^{\prime}(\cdot)}} \\
& \leq 2^{-j v} 2^{k(v+n / s)}\|b\|_{*}^{m}\|\Omega\|_{L^{s}\left(\mathbb{S}^{n-1}\right)}\left\|\chi_{B_{j}}\right\|_{L^{\tilde{p}_{1}^{\prime}(\cdot)}} \leq 2^{(k-j)(v+n / s)}\|b\|_{*}^{m}\|\Omega\|_{L^{s}\left(\mathbb{S}^{n-1}\right)}\left\|\chi_{B_{j}}\right\|_{L^{p_{1}^{\prime}(\cdot)}} .
\end{aligned}
$$

From this, we deduced

$$
\begin{array}{r}
\left\|\left[b^{m}, \mu_{\Omega}^{\rho}\right]\left(h_{j}\right)(x) \cdot \chi_{B_{k}}\right\|_{L^{p_{1}(\cdot)} \leq C} \leq \Omega\left\|_{L^{s}\left(\mathbb{S}^{n-1}\right)} 2^{-n k} 2^{(k-j)(v+n / s)}\right\| h_{j}\left\|_{L^{p_{1}(\cdot)}}\right\|\left(b(\cdot)-b_{B_{j}}\right)^{m} \cdot \chi_{B_{k}}\left\|_{L^{p_{1}(\cdot)}}\right\| \chi_{B_{j}} \|_{L^{p_{1}^{\prime}(\cdot)}} \\
+C\|\Omega\|_{L^{s}\left(\mathbb{S}^{n-1}\right)} 2^{-n k} 2^{(k-j)(v+n / s)}\|b\|_{*}^{m}\left\|h_{j}\right\|_{L^{p_{1}(\cdot)}}\left\|\chi_{B_{j}}\right\|_{L^{p_{1}^{\prime}(\cdot)}}\left\|\chi_{B_{k}}\right\|_{L^{p_{1}(\cdot)} \cdot \quad(28)}
\end{array}
$$

Applying Lemmas 1, 3, 4 and 5, we have

$$
\begin{aligned}
& \sum_{k=-\infty}^{\infty}\left\|\left(\frac{2^{k \alpha}\left|\sum_{j=-\infty}^{k-2}\left[b^{m}, \mu_{\Omega}^{\rho}\right]\left(h_{j}\right) \chi_{k}\right|}{\eta_{10}\|b\|_{*}^{m}}\right)^{q_{2}(\cdot)}\right\|\left\|_{L_{p_{2}(\cdot)}^{q_{2}(\cdot)}} \leq C \sum_{k=-\infty}^{\infty}\right\| \frac{2^{k \alpha}\left|\sum_{j=-\infty}^{k-2}\left[b^{m}, \mu_{\Omega}^{\rho}\right]\left(h_{j}\right) \chi_{k}\right|}{\eta_{10}\|b\|_{*}^{m}} \|_{L^{p_{1}(\cdot)}\left(\mathbb{R}^{n}\right)}^{\left(q_{2}^{2}\right)_{k}} \\
& \leq C \sum_{k=-\infty}^{\infty}\left(2^{k \alpha} \sum_{j=-\infty}^{k-2} 2^{-k n} 2^{(k-j)(v+n / s)}\left\|\frac{\left|h_{j}\right|}{\eta_{10}}\right\|_{L^{p_{1}(\cdot)}} \frac{1}{\|b\|_{*}^{m}}\left\|\left(b(\cdot)-b_{B_{j}}\right)^{m} \chi_{B_{k}}\right\|_{L^{p_{1}(\cdot)}}\left\|\chi_{B_{j}}\right\|_{L^{p_{1}^{\prime}(\cdot)}}\right)^{\left(q_{2}^{2}\right)_{k}}
\end{aligned}
$$




$$
\begin{aligned}
& +C \sum_{k=-\infty}^{\infty}\left(2^{k \alpha} \sum_{j=-\infty}^{k-2}(k-j)^{m} 2^{-k n} 2^{(k-j)(v+n / s)}\left\|\frac{\left|h_{j}\right|}{\eta_{10}}\right\|_{L^{p_{1}(\cdot)}}\left\|\chi_{B_{k}}\right\|_{L^{p_{1}(\cdot)}}\left\|\chi_{B_{j}}\right\|_{L^{p_{1}^{\prime}(\cdot)}}\right)^{\left(q_{2}^{2}\right)_{k}} \\
\leq & C \sum_{k=-\infty}^{\infty}\left(2^{k \alpha} \sum_{j=-\infty}^{k-2}(k-j)^{m} 2^{-k n} 2^{(k-j)(v+n / s)}\left\|\frac{\left|h_{j}\right|}{\eta_{10}}\right\|_{L^{p_{1}(\cdot)}}\left\|\chi_{B_{k}}\right\|_{L^{p_{1}(\cdot)}\left(\mathbb{R}^{n}\right)}\left\|\chi_{B_{j}}\right\|_{L^{p_{1}^{\prime}(\cdot)}}\right)^{\left(q_{2}^{2}\right)_{k}} \\
\leq & C \sum_{k=-\infty}^{\infty}\left(2^{k \alpha} \sum_{j=-\infty}^{k-2}(k-j)^{m} 2^{(k-j)(v+n / s)} 2^{-j \alpha}\left\|\frac{\left|2^{j \alpha} h \chi_{j}\right|}{\eta_{10}}\right\|_{L^{p_{1}(\cdot)}} \frac{\left\|\chi_{B_{j}}\right\|_{L_{1}^{p_{1}^{\prime}(\cdot)}}}{\left.\left\|\chi_{B_{k}}\right\|_{L^{p_{1}^{\prime}(\cdot)}}\right)^{\left(q_{2}^{2}\right)_{k}} .}\right.
\end{aligned}
$$

Now, by Lemma 2, we have

$$
\begin{aligned}
& \sum_{k=-\infty}^{\infty}\left\|\left(\frac{2^{k \alpha}\left|\sum_{j=-\infty}^{\infty}\left[b^{m}, \mu_{\Omega}^{\rho}\right]\left(h_{j}\right) \chi_{k}\right|}{\eta_{10}\|b\|_{*}}\right)^{q_{2}(\cdot)}\right\|_{L^{\frac{p_{1}(\cdot)}{q_{2}(\cdot)}}} \\
& \leq C \sum_{k=-\infty}^{\infty}\left\{\sum_{j=-\infty}^{k-2}(k-j)^{m} 2^{(k-j)\left(\alpha+v+n / s-n \delta_{2}\right)}\left\|\left(\frac{\left|2^{j \alpha} h \chi_{j}\right|}{\eta_{10}}\right)^{q_{1}(\cdot)}\right\|_{\left.L^{p_{1}(\cdot) q_{1}(\cdot)} \mathbb{R}^{n}\right)}^{\frac{1}{\left(q_{1}\right)+}}\right\}^{\left(q_{2}^{2}\right)_{k}},
\end{aligned}
$$

where

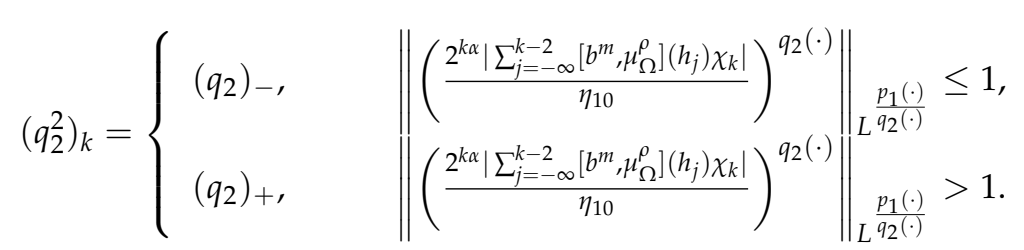

So, together with $\left(q_{1}\right)_{+}<1,\left(p_{1}\right)_{+} \leq\left(p_{2}\right)_{-} \leq\left(q_{2}^{2}\right)_{k}$, along with Remark 1, gives

$$
\begin{aligned}
& \sum_{k=-\infty}^{\infty}\left\|\left(\frac{2^{k \alpha}\left|\sum_{j=-\infty}^{k-2}\left[b^{m}, \mu_{\Omega}^{\rho}\right]\left(h_{j}\right) \chi_{k}\right|}{\eta_{10}\|b\|_{*}}\right)^{q_{2}(\cdot)}\right\|_{L_{\frac{1}{q_{2}(\cdot)}}} \sum^{\infty}\left(\sum_{j=-\infty}^{\infty}\left\|\left(\frac{\left|2^{j \alpha} h \chi_{j}\right|}{\eta_{10}}\right)^{q_{1}(\cdot)}\right\|_{L^{p_{1}(\cdot) q_{1}(\cdot)}}(k-j)^{m} 2^{(k-j)\left(\alpha+v+n / s-n \delta_{2}\right)}\right\}^{q_{*}} \\
& \leq C,
\end{aligned}
$$

where $q_{*}=\min _{k \in N} \frac{\left(q_{2}^{2}\right)_{k}}{\left(q_{1}\right)_{+}}$.

If $\left(q_{1}\right)_{+} \leq 1$, then by Hölder's inequality and Remark 1 , we have

$$
\begin{aligned}
& \sum_{k=-\infty}^{\infty}\left\|\left(\frac{2^{k \alpha}\left|\sum_{j=-\infty}^{k-2}\left[b^{m}, \mu_{\Omega}^{\rho}\right]\left(h_{j}\right) \chi_{k}\right|}{\eta_{10}\|b\|_{*}}\right)^{q_{2}(\cdot)}\right\|_{L^{\frac{p_{1}(\cdot)}{q_{2}(\cdot)}}} \leq C \sum_{k=-\infty}^{\infty}\left\{\sum_{j=-\infty}^{k-2} 2^{(k-j)\left(\alpha+v+n / s-n \delta_{2}\right)\left(q_{1}\right)_{+} / 2}\left\|\left(\frac{\left|2^{j \alpha} h \chi_{j}\right|}{\eta_{10}}\right)^{q_{1}(\cdot)}\right\|_{L^{p_{1}(\cdot) q_{1}(\cdot)}}\right\}^{\frac{\left(q_{2}^{2}\right)_{k}}{\left(q_{1}\right)+}} \\
& \times\left(\sum_{j=-\infty}^{k-2}(k-j)^{m} 2^{(k-j)\left(\alpha+v+n / s-n \delta_{2}\right)\left(\left(q_{1}\right)_{+}\right)^{\prime} / 2}\right)^{\frac{\left(q_{2}^{2}\right)_{k}}{\left(\left(q_{1}\right)+\right)^{\prime}}} \\
& \leq C\left\{\sum_{j=-\infty}^{\infty}\left\|\left(\frac{\left|2^{j \alpha} h \chi_{j}\right|}{\eta_{10}}\right)^{q_{1}(\cdot)}\right\|_{L^{p_{1}(\cdot) q_{1}(\cdot)}} \sum_{k=j+2}^{\infty} 2^{(k-j)\left(\alpha+v+n / s-n \delta_{2}\right)\left(q_{1}\right)_{+} / 2}\right\}^{q_{*}} \leq C,
\end{aligned}
$$


where $q_{*}=\min _{k \in N} \frac{\left(q_{2}^{2}\right)_{k}}{\left(q_{1}\right)_{+}}$. This implies that

$$
\eta_{21} \leq C \eta_{10}\|b\|_{*} \leq C\|b\|_{*}\|h\|_{\dot{K}_{p_{1}(\cdot)}^{\alpha, q_{1}(\cdot)}\left(\mathbb{R}^{n}\right)} .
$$

Finally we estimate $\eta_{23}$. For any $x \in A_{j}, j \geq k+2$, by the same argument as in $\eta_{21}$, we obtain

$$
\begin{aligned}
\left|\left[b^{m}, \mu_{\Omega}^{\rho}\right]\left(h_{j}\right)(x)\right| \quad:= & \left(\int_{0}^{\infty}\left|\int_{|x-y| \leq t} \frac{\Omega(x-y)}{|x-y|^{n-\rho}}[b(x)-b(y)]^{m} h_{j}(y) \mathrm{d} y\right|^{2} \frac{\mathrm{d} t}{t^{2 \rho+1}}\right)^{1 / 2} \\
\leq & \left(\int_{0}^{|y|}\left|\int_{|x-y| \leq t} \frac{\Omega(x-y)}{|x-y|^{n-\rho}}[b(x)-b(y)]^{m} h_{j}(y) \mathrm{d} y\right|^{2} \frac{\mathrm{d} t}{t^{2 \rho+1}}\right)^{1 / 2} \\
& +\left(\int_{|y|}^{\infty}\left|\int_{|x-y| \leq t} \frac{\Omega(x-y)}{|x-y|^{n-\rho}}[b(x)-b(y)]^{m} h_{j}(y) \mathrm{d} y\right|^{2} \frac{\mathrm{d} t}{t^{2 \rho+1}}\right)^{1 / 2} \\
:= & { }_{23}{ }^{\prime \prime}{ }^{\prime \prime}{ }_{23} .
\end{aligned}
$$

Noticing that $j \geq k+2$. To estimate $\eta_{23}^{\prime}$ and $\eta_{23}^{\prime \prime}$ we will use same method as that of $\eta_{21}^{\prime}$ and $\eta_{21}^{\prime \prime}$ in Step 2 . Since

$$
\begin{aligned}
{ }_{23} \leq & C 2^{(k-j) / 2} 2^{-j n}\left\{\left[b(x)-b_{B_{j}}\right]^{m}\left\|(\Omega(x-\cdot)) \cdot \chi_{j}(\cdot)\right\|_{L^{p^{(} \cdot(\cdot)}}\left\|h_{j}\right\|_{L^{p(\cdot)}}\right. \\
& \left.+\left\|\Omega(x-\cdot)\left(b_{B_{j}}-b(\cdot)\right)^{m}\left(h_{j}\right) \cdot \chi_{j}(\cdot)\right\|_{L^{p^{\prime}(\cdot)}}\left\|h_{j}\right\|_{L^{p(\cdot)}}\right\}
\end{aligned}
$$

and

$$
\begin{aligned}
{ }^{\prime}{ }_{23} \leq & C 2^{-j n}\left\{\left[b(x)-b_{B_{j}}\right]^{m}\left\|(\Omega(x-\cdot)) \cdot \chi_{j}(\cdot)\right\|_{L^{p^{\prime}(\cdot)}}\left\|h_{j}\right\|_{L^{p(\cdot)}}\right. \\
& \left.+\left\|\Omega(x-\cdot)\left(b_{B_{j}}-b(\cdot)\right)^{m}\left(h_{j}\right) \cdot \chi_{j}(\cdot)\right\|_{L^{p^{(\cdot)}}}\left\|h_{j}\right\|_{L^{p(\cdot)}}\right\} .
\end{aligned}
$$

Thus,

$$
\begin{aligned}
\left|\left[b^{m}, \mu_{\Omega}^{\rho}\right]\left(h_{j}\right)(x)\right| \leq & C 2^{-j n}\left\{\left[b(x)-b_{B_{j}}\right]^{m}\left\|(\Omega(x-\cdot)) \cdot \chi_{j}(\cdot)\right\|_{L^{p^{\prime}(\cdot)}}\left\|h_{j}\right\|_{L^{p(\cdot)}}\right. \\
& \left.+\left\|\Omega(x-\cdot)\left(b_{B_{j}}-b(\cdot)\right)^{m} \cdot \chi_{j}(\cdot)\right\|_{L^{p^{\prime}(\cdot)}}\left\|h_{j}\right\|_{L^{p(\cdot)}}\right\} .
\end{aligned}
$$

From (13), by using Lemma 7 and Lemma 2, we get

$$
\begin{aligned}
\left\|\Omega(x-\cdot)\left(b_{B_{j}}-b(\cdot)\right)^{m} \cdot \chi_{j}(\cdot)\right\|_{L^{p^{\prime}(\cdot)}} & \leq\|\Omega(x-\cdot)\|_{L^{s}}\left\|\left(b_{B_{j}}-b(\cdot)\right)^{m} \cdot \chi_{j}(\cdot)\right\|_{L^{\tilde{p}^{\prime}(\cdot)}} \\
& \leq 2^{-j v} 2^{k(v+n / s)}\|b\|_{*}^{m}\|\Omega\|_{L^{s}\left(\mathbb{S}^{n-1}\right)}\left\|\chi_{B_{j}}\right\|_{L^{\tilde{p}^{\prime}(\cdot)}} .
\end{aligned}
$$

Hence, we plug the inequality (36) into (35) and obtain

$$
\begin{aligned}
\left\|\left[b^{m}, \mu_{\Omega}^{\rho}\right]\left(h_{j}\right)(x) \chi_{B_{k}}\right\|_{L^{p_{1}(\cdot)} \leq} \leq & C\|\Omega\|_{L^{s}\left(\mathbb{S}^{n-1}\right)} 2^{-j n} 2^{(k-j)(v+n / s)}\left\|h_{j}\right\|_{L^{p_{1}(\cdot)}}\left\|\left(b(\cdot)-b_{B_{j}}\right)^{m} \chi_{B_{k}}\right\|_{L^{p_{1}(\cdot)}}\left\|\chi_{B_{j}}\right\|_{L^{p_{1}^{\prime}(\cdot)}} \\
& +C\|\Omega\|_{L^{s}\left(\mathbb{S}^{n-1}\right)} 2^{-j n} 2^{(k-j)(v+n / s)}\|b\|_{*}^{m}\left\|h_{j}\right\|_{L^{p_{1}(\cdot)}}\left\|\chi_{B_{j}}\right\|_{L^{\left.p_{1}^{(} \cdot\right)}}\left\|\chi_{B_{k}}\right\|_{L^{p_{1}(\cdot)}}
\end{aligned}
$$

By Lemma 5 and the above inequality, we have

$$
\begin{aligned}
& \sum_{k=-\infty}^{\infty}\left\|\left(\frac{2^{k \alpha}\left|\sum_{j=k+2}^{\infty}\left[b^{m}, \mu_{\Omega}^{\rho}\right]\left(h_{j}\right) \chi_{k}\right|}{\eta_{10}\|b\|_{*}^{m}}\right)^{q_{2}(\cdot)}\right\|_{L^{\frac{p_{1}(\cdot)}{q_{2}(\cdot)}}} \leq C \sum_{k=-\infty}^{\infty}\left\|\frac{2^{k \alpha}\left|\sum_{j=k+2}^{\infty}\left[b^{m}, \mu_{\Omega}^{\rho}\right]\left(h_{j}\right) \chi_{k}\right|}{\eta_{10}\|b\|_{*}^{m}}\right\|_{L^{p_{1}(\cdot)}}^{\left(q_{2}^{2}\right)_{k}} \\
& \leq C \sum_{k=-\infty}^{\infty}\left(2^{k \alpha} \sum_{j=k+2}^{\infty} 2^{-j n} 2^{(k-j)(v+n / s)}\left\|\frac{\left|h_{j}\right|}{\eta_{10}}\right\|_{L^{p_{1}(\cdot)}} \frac{1}{\|b\|_{*}^{m}}\left\|\left(b(\cdot)-b_{B_{j}}\right)^{m} \chi_{B_{k}}\right\|_{L^{p_{1}(\cdot)}}\left\|\chi_{B_{j}}\right\|_{L^{p_{1}^{\prime}(\cdot)}}\right)^{\left(q_{2}^{2}\right)_{k}}
\end{aligned}
$$




$$
\begin{aligned}
& +C \sum_{k=-\infty}^{\infty}\left(2^{k \alpha} \sum_{j=k+2}^{\infty}(j-k)^{m} 2^{-j n} 2^{(k-j)(v+n / s)}\left\|\frac{\left|h_{j}\right|}{\eta_{10}}\right\|_{L^{p_{1}(\cdot)}}\left\|\chi_{B_{k}}\right\|_{L^{p_{1}(\cdot)}}\left\|\chi_{B_{j}}\right\|_{L^{p_{1}^{\prime}(\cdot)}}\right)^{\left(q_{2}^{2}\right)_{k}} \\
\leq & C \sum_{k=-\infty}^{\infty}\left(2^{k \alpha} \sum_{j=k+2}^{\infty}(j-k)^{m} 2^{-j n} 2^{(k-j)(v+n / s)}\left\|\frac{\left|h_{j}\right|}{\eta_{10}}\right\|_{L^{p_{1}(\cdot)}}\left\|\chi_{B_{k}}\right\|_{L^{p_{1}(\cdot)}}\left\|\chi_{B_{j}}\right\|_{L^{p_{1}^{\prime}(\cdot)}}\right)^{\left(q_{2}^{2}\right)_{k}} \\
\leq & C \sum_{k=-\infty}^{\infty}\left(2^{k \alpha} \sum_{j=k+2}^{\infty}(j-k)^{m} 2^{(k-j)(v+n / s)} 2^{-j \alpha}\left\|\frac{\left|2^{j \alpha} h \chi_{j}\right|}{\eta_{10}}\right\|_{L^{p_{1}(\cdot)}} \frac{\left\|\chi_{B_{k}}\right\|_{L^{p_{1}(\cdot)}}}{\left\|\chi_{B_{j}}\right\|_{L^{p_{1}(\cdot)}}}\right)^{\left(q_{2}^{2}\right)_{k}} \\
\leq & C \sum_{k=-\infty}^{\infty}\left\{\sum_{j=k+2}^{\infty}(j-k)^{m} 2^{(k-j)\left(\alpha+v+n / s+n \delta_{12}\right)}\left\|\left(\frac{\left|2^{j \alpha} h \chi_{j}\right|}{\eta_{10}}\right)^{q_{1}(\cdot)}\right\| \|_{L^{p_{1}(\cdot) q_{1}(\cdot)}}^{\frac{1}{\left(q_{1}\right)+}}\right\}^{\left(q_{2}^{3}\right)_{k}}
\end{aligned}
$$

where

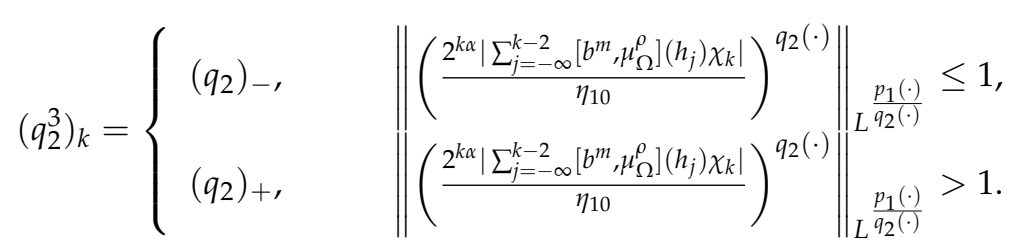

Hence, by the similar argument to Theorem 1, we arrive at $\eta_{23} \leq C \eta_{10}\|b\|_{*} \leq C\|b\|_{*}\|h\|_{\dot{K}_{p_{1}(\cdot)}^{\alpha, q_{1}(\cdot)}\left(\mathbb{R}^{n}\right)}$. This completes the proof.

Theorem 3. Let $b \in \dot{\Lambda}_{\gamma}\left(\mathbb{R}^{n}\right), 0<\gamma \leq 1, m \in \mathbb{N}, 0<\rho<n, 0<v \leq 1$. Suppose that $q_{1}^{+}<n / m \gamma, 1 / q_{1}(x)-$ $1 / q_{2}(x)=m \gamma / n, \Omega \in L^{s}\left(\mathbb{S}^{n-1}\right)\left(s>q_{2}^{+}\right)$with $1 \leq r^{\prime}<q_{2}^{-}, p_{1}(\cdot) \in \mathcal{B}\left(\mathbb{R}^{n}\right), \Omega \in L^{s}\left(\mathbb{S}^{n-1}\right), s>\left(p_{1}^{\prime}\right)_{+}$and $q_{1}(\cdot), q_{2}(\cdot) \in \mathcal{P}\left(\mathbb{R}^{n}\right)$ with $\left(q_{2}\right)_{-} \geq\left(q_{1}\right)_{+}$. If $-n \delta_{1}-v-n / s<\alpha<n \delta_{2}-v-n / s$ with $\delta_{1}, \delta_{2}$ as defined in Lemma 2 , then the operator $\left[b^{m}, \mu_{\Omega}^{\rho}\right]$ is bounded from $\dot{K}_{p_{1}(\cdot)}^{\alpha, q_{1}(\cdot)}\left(\mathbb{R}^{n}\right)$ to $\dot{K}_{p_{1}(\cdot)}^{\alpha, q_{2}(\cdot)}\left(\mathbb{R}^{n}\right.$ and from $\left(K_{p_{1}(\cdot)}^{\alpha, q_{1}(\cdot)}\left(\mathbb{R}^{n}\right)\right)$ to $\left(K_{p_{1}(\cdot)}^{\alpha, q_{2}(\cdot)}\left(\mathbb{R}^{n}\right)\right)$.

Author Contributions: All authors contributed equally to the writing of this paper. All authors read and approved the final manuscript.

Conflicts of Interest: "The authors declare no conflict of interest."

\section{References}

[1] Stein, E. M. (1958). On the functions of Littlewood-Paley, Lusin, and Marcinkiewicz. Transactions of the American Mathematical Society, 88(2), 430-466.

[2] Benedek, A., Calderön, A. P., \& Panzone, R. (1962). Convolution operators on Banach space valued functions. Proceedings of the National Academy of Sciences of the United States of America, 48(3), 356.

[3] Ding Y., On Marcinkiewicz integral, in Proceeding of the Conference Singular Integrals and Related Topics, III (Osaka 2001), 28-38.

[4] Hörmander, L. (1960). Estimates for translation invariant operators in $L^{p}$-spaces. Acta Mathematica, 104(1-2), 93-140.

[5] Walsh, T. (1972). On the function of Marcinkiewicz. Collection of articles honoring the completion by Antoni Zygmund of 50 years of scientific activity, III. Studia Mathematica, 44, 203-217.

[6] Sakamoto, M., \& Yabuta, K. (1999). Boundedness of Marcinkiewicz functions. Studia Mathematica, 2(135), $103-142$.

[7] Shi, X., \& Jiang, Y. (2009). Weighted boundedness of parametric Marcinkiewicz integral and higher order commutator. Analysis in Theory and Applications, 25(1), 25-39.

[8] Aliev, S. S., \& Guliev, V. S. (2012). Boundedness of the parametric Marcinkiewicz integral operator and its commutators on generalized Morrey spaces. Georgian Mathematical Journal, 19(2), 195-208.

[9] Al-Salman, A. (2011). On the $L^{2}$ boundedness of parametric Marcinkiewicz integral operator. Journal of Mathematical Analysis and Applications, 375(2), 745-752.

[10] Ding, Y., Lu, S., \& Yabuta, K. (2002). A problem on rough parametric Marcinkiewicz functions. Journal of the Australian Mathematical Society, 72(1), 13-22.

[11] Ding, Y., Xue, Q., \& Yabuta, K. (2012). A remark to the $L^{2}$ boundedness of parametric Marcinkiewicz integral. Journal of Mathematical Analysis and Applications, 387(2), 691-697. 
[12] Fang, Q. Q., \& Shi, X. L. (2011). Estimates for parametric Marcinkiewicz integrals in BMO and Campanato spaces. Applied Mathematics-A Journal of Chinese Universities, 26(2), 230.

[13] Huoxiong, W., \& Pu, Z. (2003). On parametric Marcinkiewicz integrals related to block spaces. Applied Mathematics-A Journal of Chinese Universities, 18(3), 258-266.

[14] Seymur S. A., \& Vagif S. G. (2012). Parametric Marcinkiewicz Integral and its commutators on generalized Morrey spaces. Georgian Mathematical Journal, 19, 195-208.

[15] Nakano, H. (1950). Modulared semi-ordered linear spaces. Maruzen Company.

[16] Musielak, J., \& Orlicz, W. (1959). On modular spaces. Studia Mathematica, 18(1), 49-65.

[17] Musielak, J. (1983). Orlicz spaces and modular spaces. Lecture notes in Mathematics, 1034, 1-216.

[18] Diening L. (2004). Maximal function on generalized Lebesgue spaces $L^{p(x)}$. Mathematical Inequalities $\mathcal{E}$ Applications, 7 , 245-253.

[19] Diening, L., \& Ruzicka, M. (2003). Calderón-Zygmund operators on generalized Lebesgue spaces $L^{p(\cdot)}$ and problems related to fluid dynamics. Journal für die reine und angewandte Mathematik, 563, 197-220.

[20] Fan, X., Shen, J., \& Zhao, D. (2001). Sobolev embedding theorems for spaces $W^{k, p(x)}(w)$, Journal of Mathematical Analysis and Applications, 262(2), 749-760.

[21] Kováčik O. \& Rákosník J. (1991). On spaces $L^{p(\cdot)}$ and $W^{k, p(\cdot)}$, Czechoslovak Mathematical Journal, 41, $592-618$.

[22] Fan, X. (2010). Variable exponent Morrey and Campanato spaces. Nonlinear Analysis: Theory, Methods E Applications, 72(11), 4148-4161.

[23] Dong, B., \& Xu, J. (2012). New Herz type Besov and Triebel-Lizorkin spaces with variable exponents. Journal of Function Spaces and Applications, 2012.

[24] Fu, J., \& Xu, J. (2011). Characterizations of Morrey type Besov and TriebelâĂŞLizorkin spaces with variable exponents. Journal of Mathematical Analysis and Applications, 381(1), 280-298.

[25] Gurka, P., Harjulehto, P., \& Nekvinda, A. (2007). Bessel potential spaces with variable exponent. Mathematical Inequalities \& Applications, 10(3), 661.

[26] Lijuan, W., \& TAO, S. (2016). Parameterized Littlewood-Paley operators and their commutators on Herz spaces with variable exponents. Turkish Journal of Mathematics, 40(1), 122-145.

[27] Capone, C., David Cruz-Uribe, S. F. O., \& Fiorenza, A. (2007). The fractional maximal operator and fractional integrals on variable $L^{p}$ spaces. Revista Matem Áatica Iberoamericana, 23(3), 743-770.

[28] Izuki, M. (2010). Boundedness of sublinear operators on Herz spaces with variable exponent and application to wavelet characterization. Analysis Mathematica, 36(1), 33-50.

[29] Izuki, M. (2010). Boundedness of commutators on Herz spaces with variable exponent. Rendiconti del Circolo Matematico di Palermo, 59(2), 199-213.

[30] Muckenhoupt, B., \& Wheeden, R. L. (1971). Weighted norm inequalities for singular and fractional integrals. Transactions of the American Mathematical Society, 161, 249-258.

[31] Nakai, E., \& Sawano, Y. (2012). Hardy spaces with variable exponents and generalized Campanato spaces. Journal of Functional Analysis, 262(9), 3665-3748.

[32] Cruz-Uribe, D. V., \& Fiorenza, A. (2013). Variable Lebesgue spaces: Foundations and harmonic analysis. Springer Science \& Business Media. 